\title{
Combining Ability Analysis for Yield and its Components in Bread Wheat (Triticum aestivum L.) under Abiotic Stress
}

\author{
Jaydev Kumar ${ }^{1}$, S.K. Singh ${ }^{1}$, Lokendra Singh ${ }^{1}$, Mukul Kumar ${ }^{2}$, \\ Meera Srivastava ${ }^{4}$, Jagbir Singh ${ }^{4}$ and Arun Kumar ${ }^{3}$ \\ ${ }^{1}$ Department of Genetics and Plant Breeding, C.S. Azad University of Agriculture and \\ Technology, Kanpur 208 002, India \\ ${ }^{2}$ Department of Botany and Plant Physiology, Mandan Bharti Agriculture College, Agwanpur, \\ Saharsa, Bihar 852 201, India \\ ${ }^{3}$ Department of Genetics and Plant Breeding, G.B. Pant University of Agriculture and \\ Technology, Pantnagar-263 145 (Uttarakhand), India \\ ${ }^{4}$ Economic Botanical Research Farm, C.S. Azad University of Agriculture and Technology, \\ Nawabganj, Kanpur 208 002, India \\ *Corresponding author
}

\section{A B S T R A C T}

\section{Keywords}

Bread wheat, Combining ability, Gene effects, Yield components.

Article Info

Accepted: 08 February 2017 Available Online: 10 March 2017
Combining ability analysis for yield and its component under late sown condition in bread wheat involved ten diverse parents and their $45 \mathrm{~F}_{1} \mathrm{~s}$ and their $\mathrm{F}_{2} \mathrm{~s}$ indicated significance differences among the parents for gca and crosses for sca for all characters under studied. The GCA and SCA components of variances in both generations showed significant for all traits indicating additive and non additive gene action controlled the pattern of inheritance for the concern traits over the both generations. Based on the general combining ability effects and per se performance, parent K 0307 and K 0911 emerged as good general combiners for grain yield and average to high combiners for almost of the yield component characters in late sown condition, it means these genotypes probably possessed the desirable genes for heat temperature during reproductive phase. Whereas K 0307 showed also good general combiner as their gca effect as well as per se performance for number of spikelets, number of grains per spike, grain weight per spike, spike length, 1000 grain weight while K 0911 exhibited good general combiner for protein content based on per se performance and GCA effect in both generations. On the basis on per se performance and sca effects, DBW 14 x K 0424 and K 9533 x K 0307 possessed good super combinations for grain yield and its related components whereas, K 0607 x K 0307 exhibited good cross combination for protein content based on their per se performance in both generations. The good cross combinations were the product of high $\mathrm{x}$ high, high $\mathrm{x}$ low or low $\mathrm{x}$ low general combiners. Hybridization scheme for wheat improvement, such as multiple crossing or biparental mating could be useful in further manipulation of genes for economic purposes.

\section{Introduction}

With rice and maize, wheat is essential for human cultivation. With more than 215 million hectares planted annually, wheat is the most widely cultivated cereal in the world. It 
is the most important source of dietary protein and provides around $20 \%$ of the global calories for human consumption. With around 130 million tonnes, annual global wheat trade is higher than that of maize and rice combined. More than $60 \%$ of wheat is produced in emerging and developing countries; both China and India together produce nearly twice as much as wheat as the USA and Russia combined.

Among the major staples, wheat is the only crop adapted to low temperatures that can be grown during the cool season and drought tolerant crop among cereals. But unfortunately it is also the most sensitive to high night and day temperatures. Wheat yield model indicate that a $1^{0} \mathrm{c}$ temperature increase reduces yield potential of wheat $10 \%$ in some part of world. Expert from the Intergovernmental Penal on Climate Change (IPCC) report that an average temperature increase of $1.5-6^{0} \mathrm{C}$ by the end of this country is likely and the World Bank estimate that we are barreling down a path to heat up by $4^{0} \mathrm{c}$ if the problem of climate change is not tackled aggressively now. The world leading wheat belts, wheat yield in 2050 could decline down to $27 \%$ compared to 2000 by scientist project 2050. So wheat production needs to be increase by around $60 \%$ by 2050 to meet the demand of a growing population with a challenging diet, the challenges of wheat breeders termed. So, breeder interests to development of new wheat varieties expressed their better response under heat regions.

For advancement in the yield of wheat requires certain information regarding the nature of combining ability of parents available for use in the hybridization programme of some quantitative traits have economic importance. Information of general and specific combining ability effects is very important in making the next phase of a breeding programme for heat tolerance. Many workers have reported GCA and SCA effects for yield and its component in wheat (Dubey et al., 2001; Wahid et al., 2007; Kapoor et al., 2011; Ankita et al., 2012). Through, diallel cross analysis a number of parental lines can be tested in all possible combinations. Thus the main objective of the present study was to identify the best combiners and their crosses on the basis of their general and specific combining ability for yield and its components under late sown condition.

\section{Materials and Methods}

Ten diverse parents of bread wheat (Triticum aestivum L.) i.e., K 9533, K 9162, K 1114, DBW 14, K 0607, K 0424, K 0911, K 0307, NW 2036 and K 9423 were selected on the basis of a broad range of diversity for major yield and its component characters under heat tolerance condition. The experiment was conducted during Rabi 2014-15 at Crop Research Farm (Nawabganj) of C.S. Azad University of Agriculture and Technology, Kanpur, Uttar Pradesh. The experimental materials was comprised of 100 genotypes including 10 parents and their resulting $45 \mathrm{~F}_{1} \mathrm{~s}$ and $45 \mathrm{~F}_{2} \mathrm{~S}$ in a randomized complete block design with three replication under late sown condition. In each replication parents and their $F_{1} S$ and $F_{2} S$ were randomly assigned to experimental unit/plots. Each plot comprised single row of $4 \mathrm{~m}$ with spacing of $20 \mathrm{~cm}$ between rows. Seed was planted at $5 \mathrm{~cm}$ apart. All recommended cultural practices were applied to raise a good crop. Five competitive plants in parents and their $\mathrm{F}_{1}$, $\mathrm{S}$ and ten plants in $\mathrm{F}_{2}$,s progenies were selected randomly from each replication for recording observation on eleven yield and its component traits viz., number of effective tillers per plant, number of spikelets per spike, number of grains per spike, grain weight per spike $(\mathrm{g})$, spike length $(\mathrm{cm})$, biological yield per plant (g), harvest index (\%), 1000 grain 
weight $(\mathrm{g})$, spike density, protein content (\%) and grain yield per plant $(\mathrm{g})$. The mean of each plot was used for statistical analysis. The combining analysis was computed according to Griffings, 1956.

\section{Results and Discussions}

Analysis of variance for combining ability revealed that the variance due to general combining ability ( $g c a)$ and specific combining ability ( $s c a$ ) were highly significant for all the characters under studied over both generations given in table 1(a) and 1(b). Thus the both kind of gene effects pictured important in controlling the pattern of inheritance of all the characters under studied. The $(\mathrm{gca} / \mathrm{sca})^{0.5}$ variance ratio was below unity in both generations for all the characters indicating the preponderance of additive gene effects present in both generations for all traits under studied. The similar findings were reported by Vanpariya et al., (2006) for different characters. Due to differences in the experimental and condition which evaluation is done some differences in the reports i.e., grain yield and some others components governed by both additive and non additive gene effects. Though, variances of specific combining ability were most pronounced than variances of general combining ability for all characters under studied. The prepotencies of non additive genetic variance for difference characters indicating that the best cross combinations could be selected on the basis on sca for further substantial.

On the basis on general combining ability effects and per se performance (table 2 and table 4), it showed that among parents, Parents K 0307 was best general combiner for number of spikelets per spike, number of grains per spike, grain weight per spike $(\mathrm{g})$, spike length $(\mathrm{cm}), 1000$ grain weight $(\mathrm{g})$, and grain yield per plant (g) while, parent K 0911 was good general combiner for protein content $(\%)$ and grain yield per plant and $\mathrm{K}$
9423, K 0307 for high biological yield per plant over both generations. Although, on the basis on general combining ability effects, parents K 0607 for spike length $(\mathrm{cm}), 1000$ grain weight $(\mathrm{g})$, protein content $(\%), \mathrm{K} 1114$ for lowest biological yield per plant $(\mathrm{g})$, harvest index (\%), DBW 14 and K 9423 for spike density, and NW 2036 for protein content $(\%)$ were identified good general combiners over both generations. Therefore, these parents have good potential and can be utilized in synthesizing a dynamic population with most desirable genes accumulated. Apparently, thus, there is still further scope for improving upon the combining ability for component traits, as none of the higher combiner for grain yield was a higher combiner or at least an average combiner for all the desirable traits. In bread wheat, parents having good general combining ability have been reported by Desai et al., (2005); Bikram and Ahmed, (2008); Ajmal et al., (2011) and Ankita et al., (2012). It was observed that top parents on the basis of high per se performance also have high general combining ability effects. Since, gca effects are attributed to additive and additive $\mathrm{x}$ additive gene effects, the above mentioned parents for gca effects have good potential for respective traits and may be used in multiple crossing breeding programme to isolate a imaginable population with desired gene manipulated of grain yield. It seems practicable, that the gca rank for grain yield is related to the useful yield components, it is therefore, recommended the breeder should breed for superior combining ability for the component traits with a ultimate objective to improve the overall gca for grain yield in bread wheat. The good parents having desirable gca effects for grain yield per plant in different generations revealed that the gca effects and per se performance were positively correlated in most of the best parents but few cases is not allow such conditions. 
Table.1(a) Analysis of variance for combining ability in a 10 parent- diallel cross (parents and their F1s) among 11th characters in bread wheat

\begin{tabular}{|c|c|c|c|c|c|c|c|c|c|c|c|c|}
\hline $\begin{array}{l}\text { Source of } \\
\text { variation }\end{array}$ & d.f & $\begin{array}{l}\text { No. of } \\
\text { effective } \\
\text { tillers per } \\
\text { plant }\end{array}$ & $\begin{array}{l}\text { No. of } \\
\text { spikelets } \\
\text { per spike }\end{array}$ & $\begin{array}{l}\text { No. of } \\
\text { grains per } \\
\text { spike }\end{array}$ & $\begin{array}{l}\text { Grain } \\
\text { weight per } \\
\text { spike (g) }\end{array}$ & $\begin{array}{l}\text { Spike } \\
\text { length }(\mathbf{c m})\end{array}$ & $\begin{array}{l}\text { Biological } \\
\text { yield per } \\
\text { plant (g) }\end{array}$ & $\begin{array}{l}\text { Harvest } \\
\text { index }(\%)\end{array}$ & $\begin{array}{l}1000 \text { grain } \\
\text { weight (g) }\end{array}$ & $\begin{array}{l}\text { Spike } \\
\text { density }\end{array}$ & $\begin{array}{l}\text { Protein } \\
\text { content } \\
(\%)\end{array}$ & $\begin{array}{l}\text { Grain yield } \\
\text { per plant } \\
\text { (g) }\end{array}$ \\
\hline GCA & 9 & $0.68^{* *}$ & $1.45^{* *}$ & 53.29 ** & $0.12^{* * *}$ & $1.11^{* *}$ & $17.68^{* *}$ & $20.11 * *$ & $7.93 * *$ & $0.01419 * *$ & $3.95^{* *}$ & $6.47 * *$ \\
\hline SCA & 45 & $0.82 * *$ & $2.34 * *$ & $43.58^{* *}$ & $0.13^{* *} *$ & $0.51^{* *}$ & $17.33^{* *}$ & $36.14 * *$ & $4.34 * *$ & $0.01155^{* *}$ & $1.42 * *$ & $7.77 * *$ \\
\hline Error & 108 & 0.05 & 0.26 & 6.82 & 0.006 & 0.013 & 0.18 & 1.34 & 0.12 & 0.00209 & 0.02 & 0.12 \\
\hline$\sigma^{2} \mathrm{~g}$ & & 0.05248 & 0.09929 & 3.97245 & 0.00948 & 0.09087 & 1.45800 & 1.56330 & 0.65087 & 0.00101 & 0.32843 & 0.52970 \\
\hline$\sigma^{2} s$ & & 0.77202 & 2.08119 & 36.76074 & 0.12375 & 0.49893 & 17.14829 & 34.79825 & 4.22376 & 0.00946 & 1.41359 & 7.65193 \\
\hline$\left(\sigma^{2} g / \sigma^{2} s\right)^{0.5}$ & & 0.2607 & 0.2184 & 0.3245 & 0.2768 & 0.4267 & 0.2915 & 0.2119 & 0.3925 & 0.3265 & 0.4820 & 0.2630 \\
\hline
\end{tabular}

Table.1(b) Analysis of variance for combining ability in a 10 parent- diallel cross (parents and their F2s) among 11th characters in bread wheat

\begin{tabular}{|c|c|c|c|c|c|c|c|c|c|c|c|c|}
\hline $\begin{array}{l}\text { Source of } \\
\text { variation }\end{array}$ & $d . f$. & $\begin{array}{l}\text { No. of } \\
\text { effective } \\
\text { tiller per } \\
\text { plant }\end{array}$ & $\begin{array}{l}\text { No. of } \\
\text { spikelets } \\
\text { per spike }\end{array}$ & $\begin{array}{l}\text { No. of } \\
\text { grains per } \\
\text { spike }\end{array}$ & $\begin{array}{l}\text { Grain } \\
\text { weight per } \\
\text { Spike (g) }\end{array}$ & $\begin{array}{l}\text { Spike } \\
\text { length } \\
\text { (cm) }\end{array}$ & $\begin{array}{l}\text { Biological } \\
\text { yield per } \\
\text { Plant (g) }\end{array}$ & $\begin{array}{l}\text { Harvest } \\
\text { index }(\%)\end{array}$ & $\begin{array}{l}1000 \text { grain } \\
\text { weight (g) }\end{array}$ & $\begin{array}{l}\text { Spike } \\
\text { density }\end{array}$ & $\begin{array}{l}\text { Protein } \\
\text { content } \\
(\%)\end{array}$ & $\begin{array}{l}\text { Grain } \\
\text { yield per } \\
\text { plant (g) }\end{array}$ \\
\hline GCA & 9 & $0.52 * *$ & $0.75^{* *}$ & $32.36^{* * *}$ & $0.09 * *$ & $0.96^{* * *}$ & $6.58 * *$ & $19.01 * *$ & $5.02 * *$ & 0.01582 & $1.64 * *$ & $1.33 * *$ \\
\hline SCA & 45 & $0.38 * *$ & $0.90^{* *}$ & $34.76^{* *}$ & $0.06^{* * *}$ & $0.35^{* *}$ & $8.12 * *$ & $15.83^{* *}$ & $3.00 * *$ & 0.01039 & $0.73^{* *}$ & $1.89 * *$ \\
\hline Error & 108 & 0.04 & 0.07 & 7.33 & 0.009 & 0.03 & 0.43 & 3.85 & 0.17 & 0.00147 & 0.04 & 0.18 \\
\hline$\sigma^{2} \mathrm{~g}$ & & 0.04088 & 0.05650 & 2.08586 & 0.00676 & 0.07754 & 0.51274 & 1.26338 & 0.40444 & 0.00120 & 0.13383 & 0.09595 \\
\hline$\sigma^{2} s$ & & 0.35166 & 0.83120 & 27.42676 & 0.05583 & 0.32426 & 7.69504 & 11.97968 & 2.82960 & 0.00892 & 0.70206 & 1.71779 \\
\hline$\left(\sigma^{2} g / \sigma^{2} s\right)^{0.5}$ & & 0.3409 & 0.2607 & 0.2757 & 0.3348 & 0.4890 & 0.2581 & 0.3247 & 0.3780 & 0.3661 & 0.4366 & 0.2363 \\
\hline
\end{tabular}

Note: * significant at $\mathrm{p}=0.05$ and $* *$ significant at $\mathrm{p}=0.01$ 
Table.2 Estimates of mean performance and their GCA effect of 10 diallel parents for 11th characters in bread wheat

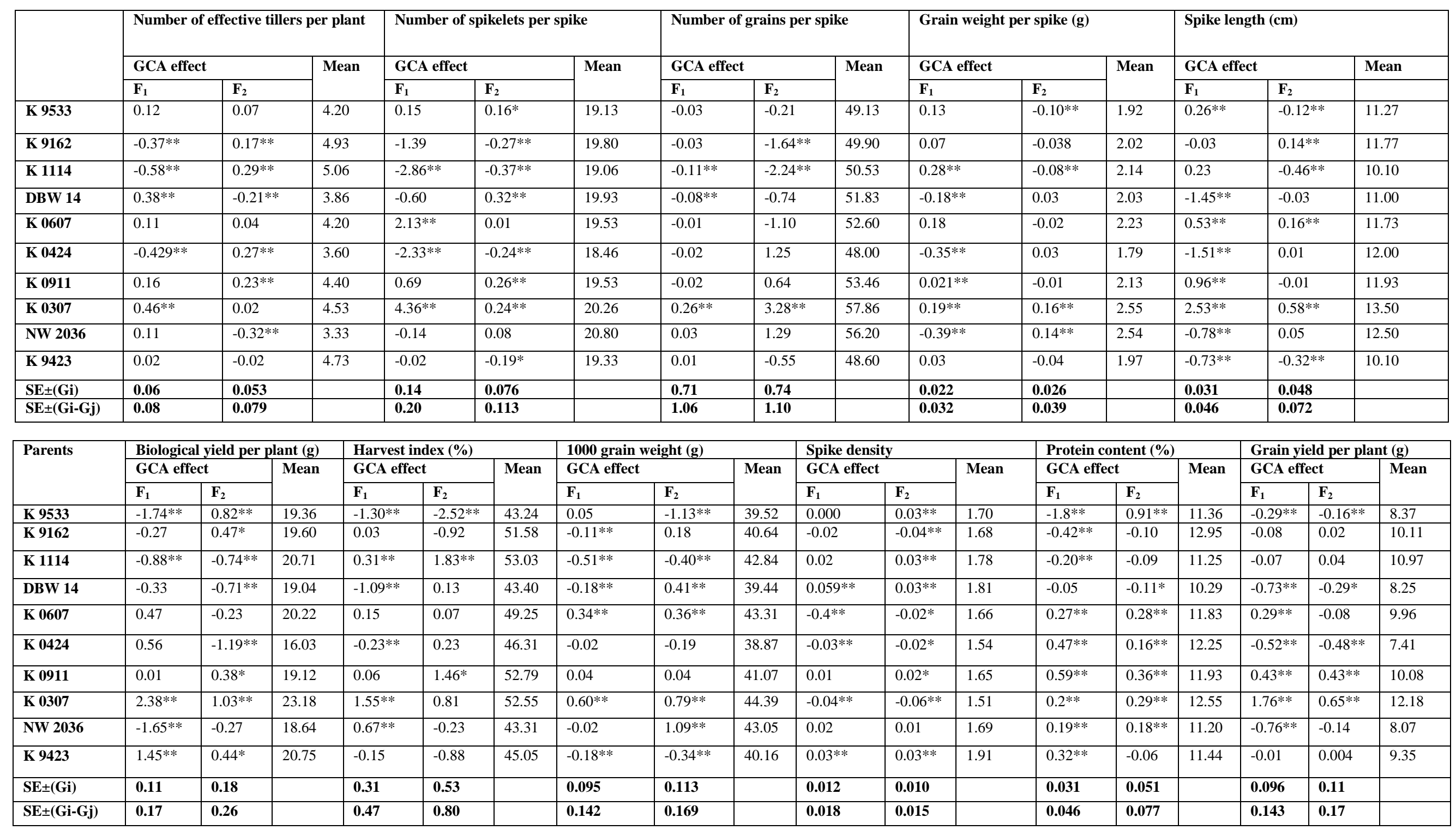


Table.3 Estimates of specific combining effects and corresponding mean performance of $45 \mathrm{~F} 1 \mathrm{~s}$ and $45 \mathrm{~F} 2 \mathrm{~s}$ for 11 th characters in bread wheat

\begin{tabular}{|c|c|c|c|c|c|c|c|c|}
\hline \multirow[t]{3}{*}{ Crosses } & \multicolumn{4}{|c|}{ No. of effective tillers per plant } & \multicolumn{4}{|c|}{ No. of spikelets per spike } \\
\hline & \multicolumn{2}{|c|}{$\mathbf{F}_{1}$} & \multicolumn{2}{|c|}{$\mathbf{F}_{2}$} & \multicolumn{2}{|c|}{$\mathbf{F}_{1}$} & \multicolumn{2}{|c|}{$\mathbf{F}_{2}$} \\
\hline & $\begin{array}{c}S C A \\
\text { effect }\end{array}$ & Mean & $\begin{array}{c}S C A \\
\text { effect }\end{array}$ & Mean & $\begin{array}{c}S C A \\
\text { effect }\end{array}$ & Mean & $\begin{array}{c}S C A \\
\text { effect }\end{array}$ & Mean \\
\hline $1 . \mathrm{K} 9533 \times \mathrm{K} 9162$ & 0.04 & 5.00 & 0.14 & 4.80 & $1.46^{* *}$ & 21.00 & 0.25 & 19.67 \\
\hline 2.K $9533 \times$ X 1114 & 0.16 & 5.33 & $-0.52 * *$ & 4.27 & $2.67 * *$ & 22.00 & $1.56 * *$ & 20.87 \\
\hline 3. K $9533 \times$ x DW 14 & $-0.44 *$ & 4.27 & -0.35 & 3.93 & -0.63 & 19.67 & $-0.74 * *$ & 19.27 \\
\hline 4.K $9533 \times$ × K 0607 & $0.86^{* *}$ & 5.93 & $0.74 * *$ & 5.27 & 0.59 & 20.60 & 0.18 & 19.87 \\
\hline $5 . \mathrm{K} 9533 \times \mathrm{K} 0424$ & -0.28 & 4.27 & $-0.68 * *$ & 3.53 & $-3.75^{* *}$ & 15.73 & 0.22 & 19.67 \\
\hline $6 . \mathrm{K} 9533 \times \mathrm{K} 0911$ & -0.16 & 4.93 & 0.35 & 5.07 & $1.19^{*}$ & 21.27 & $0.53^{*}$ & 20.47 \\
\hline 7. K $9533 \times \mathrm{K} 0307$ & $0.91^{* *}$ & 6.00 & $0.83 * *$ & 5.33 & 0.62 & 21.00 & 0.15 & 20.07 \\
\hline 8. K 9533 x NW 2036 & -0.30 & 4.20 & $-0.57 * *$ & 3.60 & -0.09 & 19.93 & -0.37 & 19.40 \\
\hline 9. K $9533 \times$ K 9423 & $0.60 * *$ & 5.47 & $0.79 * *$ & 5.27 & -0.27 & 19.67 & -0.35 & 19.13 \\
\hline 10. K 9162 x K 1114 & $-0.90^{* *}$ & 4.33 & $-0.94 * *$ & 3.93 & $-1.30^{* *}$ & 17.53 & $-0.54 *$ & 18.33 \\
\hline 11.K 9162 x DBW 14 & $-0.43^{*}$ & 4.33 & $-0.57 * *$ & 3.80 & $-2.80 * *$ & 17.00 & $-2.17 * *$ & 17.40 \\
\hline 12. K $9162 \times$ K 0607 & 0.07 & 5.20 & $0.38^{*}$ & 5.00 & 0.28 & 19.80 & 0.41 & 19.67 \\
\hline 13. K 9162 × K 0424 & $0.60 * *$ & 5.20 & 0.10 & 4.40 & 0.81 & 19.80 & 0.12 & 19.13 \\
\hline 14. K 9162 x K 0911 & -0.23 & 4.93 & -0.21 & 4.60 & -0.58 & 19.00 & -0.11 & 19.40 \\
\hline 15. K9162 x K 0307 & $0.59^{* *}$ & 5.73 & $0.54 * *$ & 5.13 & 0.05 & 19.93 & -0.42 & 19.07 \\
\hline 16. K $9162 \times$ NW 2036 & $-0.89^{* * *}$ & 3.67 & $-0.53^{* *}$ & 3.73 & -0.73 & 18.80 & 0.26 & 19.60 \\
\hline 17. K 9162 x K 9423 & $1.34 * *$ & 6.27 & $0.70 * *$ & 5.27 & $1.29 * *$ & 20.73 & $0.55^{*}$ & 19.60 \\
\hline 18. K $1114 \times$ x DBW 14 & $-0.64 * *$ & 4.33 & $-0.36^{*}$ & 4.13 & -0.06 & 19.53 & $0.87 * *$ & 20.33 \\
\hline 19. K 1114 x K 0607 & $-0.94 * *$ & 4.40 & 0.25 & 5.00 & $-3.51 * *$ & 15.80 & $1.85^{* * *}$ & 21.00 \\
\hline 20. K 1114 x K 0424 & $0.86^{* * *}$ & 5.67 & -0.10 & 4.33 & $3.69^{* *}$ & 22.47 & $-1.77 * *$ & 17.13 \\
\hline 21. K 1114 x K 0911 & $2.64 * *$ & 8.00 & $1.93 * *$ & 6.87 & 0.56 & 19.93 & 0.40 & 19.80 \\
\hline 22. K 1114 x K 0307 & $-0.75^{* *}$ & 4.60 & $-0.39 *$ & 4.33 & -0.88 & 18.80 & 0.02 & 19.40 \\
\hline 23. K 1114 x NW 2036 & $2.04 * *$ & 6.80 & $0.94 * *$ & 5.33 & -0.05 & 19.27 & -0.23 & 19.00 \\
\hline 24. K 1114 x K 9423 & $-1.73^{* *}$ & 3.40 & $-0.96 * *$ & 3.73 & 0.03 & 19.27 & $-0.75 * *$ & 18.20 \\
\hline 25. DBW 14 x K 0607 & $0.93 * *$ & 5.80 & $0.76 * *$ & 5.00 & $1.79 * *$ & 22.07 & 0.09 & 19.93 \\
\hline 26. DBW 14 x K 0424 & $1.92 * *$ & 6.27 & $1.08 * *$ & 5.00 & $2.32 * *$ & 22.07 & $1.13 * *$ & 20.73 \\
\hline 27. DBW 14 x K 0911 & $-0.63 * *$ & 4.27 & $-0.43^{*}$ & 4.00 & -0.54 & 19.80 & $-0.70 * *$ & 19.40 \\
\hline 28. DBW 14 x K 0307 & -0.09 & 4.80 & -0.22 & 4.00 & 0.36 & 21.00 & $0.86^{* * *}$ & 20.93 \\
\hline 29. DBW $14 \times$ NW 2036 & 0.04 & 4.33 & $0.78 * *$ & 4.67 & 0.71 & 21.00 & $0.94 * *$ & 20.87 \\
\hline 30. DBW 14 x K 9423 & $0.60 * *$ & 5.27 & $-0.45^{*}$ & 3.73 & 0.13 & 20.33 & 0.16 & 19.80 \\
\hline 31. K 0607 x K 0424 & $0.56^{* *}$ & 5.27 & 0.09 & 4.27 & 0.74 & 20.20 & $1.78 * *$ & 21.07 \\
\hline 32. K 0607 x K 0911 & -0.20 & 5.07 & $-0.61 * *$ & 4.07 & $2.27 * *$ & 22.33 & -0.25 & 19.53 \\
\hline 33. K 0607 x K 0307 & $0.55^{* *}$ & 5.80 & $-0.67 * *$ & 3.80 & -0.03 & 20.33 & $-1.10 * *$ & 18.67 \\
\hline 34. K 0607 x NW 2036 & $0.47 *$ & 5.13 & 0.07 & 4.20 & $-1.60^{* * *}$ & 18.40 & $-2.88 * *$ & 16.73 \\
\hline 35. K 0607 x K 9423 & -0.23 & 4.80 & $-0.43^{*}$ & 4.00 & 0.41 & 20.33 & -0.06 & 19.27 \\
\hline 36. K 0424 x K 0911 & $-1.07 * *$ & 3.67 & 0.10 & 4.47 & $-2.93 * *$ & 16.60 & -0.01 & 19.53 \\
\hline 37. K 0424 x K 0307 & -0.39 & 4.33 & -0.22 & 3.93 & -0.83 & 19.00 & $-0.65^{*}$ & 18.87 \\
\hline 38. K 0424 x NW 2036 & $-0.67 * *$ & 3.47 & 0.32 & 4.13 & -0.47 & 19.00 & -0.37 & 19.00 \\
\hline 39. K 0424 x K 9423 & -0.37 & 4.13 & -0.18 & 3.93 & $1.35 * *$ & 20.73 & $0.71 * *$ & 19.80 \\
\hline 40. K 0911 x K 0307 & 0.39 & 5.67 & $-0.59 * *$ & 4.07 & 0.58 & 21.00 & -0.08 & 19.93 \\
\hline 41. K 0911 x NW 2036 & $0.84 * *$ & 5.53 & 0.01 & 4.33 & $1.60 * *$ & 21.67 & $0.74 * *$ & 20.60 \\
\hline 42. K 0911 x K 9423 & 0.21 & 5.27 & $0.38^{*}$ & 5.00 & $-0.98 *$ & 19.00 & 0.49 & 20.07 \\
\hline 43. K 0307 x NW 2036 & 0.25 & 4.93 & $0.49 * *$ & 4.60 & 0.50 & 20.87 & $0.76^{* *}$ & 20.60 \\
\hline 44. K 0307 x K 9423 & 0.02 & 5.07 & 0.05 & 4.47 & 0.52 & 20.80 & -0.09 & 19.47 \\
\hline 45. NW 2036 x K 9423 & -0.26 & 4.20 & $-0.61 * *$ & 3.47 & $-1.46^{* *}$ & 18.47 & $-1.07 * *$ & 18.33 \\
\hline $\mathrm{SE} \pm(\mathrm{Sij})$ & \multicolumn{2}{|l|}{0.20} & \multicolumn{2}{|l|}{0.17} & \multicolumn{2}{|l|}{0.47} & \multicolumn{2}{|l|}{0.25} \\
\hline $\mathrm{SE} \pm($ Sij-Sik) & \multicolumn{2}{|l|}{0.29} & \multicolumn{2}{|l|}{0.26} & \multicolumn{2}{|l|}{0.69} & \multicolumn{2}{|l|}{0.37} \\
\hline
\end{tabular}


Int.J.Curr.Microbiol.App.Sci (2017) 6(3): 24-39

Table. 3 continued

\begin{tabular}{|c|c|c|c|c|c|c|c|c|}
\hline \multirow[t]{3}{*}{ Crosses } & \multicolumn{4}{|c|}{ No. of grains per spike } & \multicolumn{4}{|c|}{ Grain weight per spike (g) } \\
\hline & \multicolumn{2}{|c|}{$\mathbf{F}_{1}$} & \multicolumn{2}{|c|}{$\mathbf{F}_{2}$} & \multicolumn{2}{|c|}{$\mathrm{F}_{1}$} & \multicolumn{2}{|c|}{$\mathbf{F}_{2}$} \\
\hline & $\begin{array}{c}S C A \\
\text { effect }\end{array}$ & Mean & $\begin{array}{c}S C A \\
\text { effect }\end{array}$ & Mean & $\begin{array}{c}S C A \\
\text { effect }\end{array}$ & Mean & $\begin{array}{c}S C A \\
\text { effect }\end{array}$ & Mean \\
\hline $1 . \mathrm{K} 9533 \times \mathrm{K} 9162$ & 3.64 & 55.13 & -3.03 & 43.40 & 0.13 & 2.22 & -0.11 & 1.71 \\
\hline $2 . \mathrm{K} 9533 \times \mathrm{K} 1114$ & 2.54 & 52.57 & 1.63 & 47.47 & 0.11 & 2.12 & -0.15 & 1.63 \\
\hline 3. K $9533 \times$ DBW 14 & -3.05 & 49.23 & $-6.20^{*}$ & 41.13 & -0.12 & 1.92 & -0.17 & 1.66 \\
\hline 4.K 9533 x K 0607 & 1.45 & 56.47 & 2.30 & 49.27 & $0.23 * *$ & 2.33 & 0.00 & 1.84 \\
\hline $5 . \mathrm{K} 9533 \times \mathrm{K} 0424$ & 0.11 & 50.67 & -2.13 & 47.20 & -0.12 & 1.98 & -0.13 & 1.75 \\
\hline $6 . \mathrm{K} 9533 \times \mathrm{K} 0911$ & 3.89 & 57.47 & 1.55 & 50.27 & $0.21 * *$ & 2.31 & 0.12 & 1.97 \\
\hline 7. K $9533 \times \mathrm{K} 0307$ & $5.35^{*}$ & 62.60 & $6.44 *$ & 57.80 & $0.31 * *$ & 2.69 & $0.25 * *$ & 2.27 \\
\hline 8. K 9533 x NW 2036 & -3.61 & 49.13 & $-6.70^{*}$ & 42.67 & $-0.26 * *$ & 1.88 & $-0.28 * *$ & 1.72 \\
\hline 9. K $9533 \times$ K 9423 & -2.53 & 50.33 & 3.61 & 51.13 & $-0.17 *$ & 1.96 & 0.15 & 1.96 \\
\hline 10. K 9162 x K 1114 & -3.48 & 45.00 & -4.54 & 39.87 & $-0.18^{*}$ & 1.83 & -0.09 & 1.75 \\
\hline 11.K 9162 x DBW 14 & $-5.20^{*}$ & 45.53 & 1.10 & 47.00 & $-0.29 * *$ & 1.75 & -0.09 & 1.80 \\
\hline 12. K $9162 \times$ K 0607 & -4.20 & 49.27 & 0.13 & 45.67 & -0.01 & 2.10 & 0.08 & 1.99 \\
\hline 13. K 9162 x K 0424 & $7.25 * *$ & 56.27 & -1.23 & 46.67 & 0.14 & 2.24 & -0.04 & 1.91 \\
\hline 14. K 9162 x K 0911 & -1.77 & 50.27 & 1.78 & 49.07 & -0.09 & 2.01 & 0.07 & 1.99 \\
\hline 15. K9162 x K 0307 & -0.97 & 54.73 & $-5.26^{*}$ & 44.67 & 0.11 & 2.49 & $-0.26 * *$ & 1.82 \\
\hline 16. K 9162 x NW 2036 & $-8.46^{* * *}$ & 42.73 & 0.00 & 47.93 & $-0.42 * *$ & 1.73 & 0.02 & 2.08 \\
\hline 17. K 9162 x K 9423 & $13.28^{* * *}$ & 64.60 & 1.24 & 47.33 & $0.73 * *$ & 2.86 & 0.13 & 2.01 \\
\hline 18. K $1114 \times$ x DBW 14 & 3.26 & 52.53 & -1.24 & 44.07 & 0.13 & 2.09 & 0.01 & 1.85 \\
\hline 19. K 1114 x K 0607 & $14.73 * *$ & 66.73 & -4.41 & 40.53 & $-0.58 * *$ & 1.45 & -0.15 & 1.70 \\
\hline 20. K 1114 x K 0424 & $-8.61 * *$ & 38.93 & $6.77 * *$ & 54.07 & $0.76^{* *}$ & 2.78 & 0.01 & 1.92 \\
\hline 21. K 1114 x K 0911 & -4.70 & 45.87 & $-7.76^{* * *}$ & 38.93 & $-0.29 * *$ & 1.73 & $-0.34 * *$ & 1.53 \\
\hline 22. K 1114 x K 0307 & $-5.04 *$ & 49.20 & 1.13 & 50.47 & $-0.29 * *$ & 2.01 & 0.08 & 2.12 \\
\hline 23. K 1114 x NW 2036 & 1.74 & 51.47 & -2.68 & 44.67 & $0.29 * *$ & 2.36 & -0.04 & 1.98 \\
\hline 24. K 1114 x K 9423 & $-7.46^{* *}$ & 42.40 & -2.37 & 43.13 & $-0.38 * *$ & 1.67 & -0.03 & 1.79 \\
\hline 25. DBW 14 x K 0607 & 0.81 & 55.07 & $6.23 *$ & 52.67 & 0.00 & 2.06 & 0.12 & 2.02 \\
\hline 26. DBW 14 x K 0424 & $14.80 * *$ & 64.60 & $6.21 *$ & 55.00 & $0.85 * *$ & 2.90 & $0.51^{* *}$ & 2.46 \\
\hline 27. DBW 14 x K 0911 & $-11.43 * *$ & 41.40 & $-10.92 * *$ & 37.27 & $-0.34 * *$ & 1.71 & $-0.46 * *$ & 1.46 \\
\hline 28. DBW 14 x K 0307 & -4.29 & 52.20 & $-8.23 * *$ & 42.60 & $-0.38^{* * *}$ & 1.96 & $-0.28 * *$ & 1.81 \\
\hline 29. DBW 14 x NW 2036 & -3.92 & 48.07 & 0.70 & 49.53 & -0.10 & 2.00 & 0.05 & 2.12 \\
\hline 30. DBW 14 x K 9423 & $8.42 * *$ & 60.53 & 2.27 & 49.27 & $0.16^{*}$ & 2.24 & 0.03 & 1.91 \\
\hline 31. K 0607 x K 0424 & -3.53 & 49.00 & 4.04 & 52.47 & $-0.20 * *$ & 1.92 & $0.28 * *$ & 2.25 \\
\hline 32. K 0607 x K 0911 & $9.78 * *$ & 65.33 & 4.68 & 52.50 & $0.78^{* *}$ & 2.90 & $0.29 * *$ & 2.23 \\
\hline 33. K 0607 x K 0307 & 0.38 & 59.60 & -4.33 & 46.13 & -0.02 & 2.38 & $-0.20 *$ & 1.90 \\
\hline 34. K 0607 x NW 2036 & 0.35 & 55.07 & $-10.61 * *$ & 37.87 & -0.13 & 2.04 & $-0.57 * *$ & 1.51 \\
\hline 35. K 0607 x K 9423 & $-10.98 * *$ & 43.87 & $-11.10 * *$ & 35.53 & $-0.27 * *$ & 1.88 & $-0.46 * *$ & 1.43 \\
\hline 36. K 0424 x K 0911 & $-9.63 * *$ & 41.47 & $-5.85^{*}$ & 44.33 & $-0.81 * *$ & 1.30 & $-0.30 * *$ & 1.68 \\
\hline 37. K 0424 x K 0307 & -0.43 & 54.33 & -0.82 & 52.00 & -0.10 & 2.29 & 0.00 & 2.15 \\
\hline 38. K 0424 x NW 2036 & -1.66 & 48.60 & -0.83 & 50.00 & 0.02 & 2.18 & 0.09 & 2.22 \\
\hline 39. K 0424 x K 9423 & 1.88 & 52.27 & -0.59 & 48.40 & 0.11 & 2.25 & 0.02 & 1.96 \\
\hline 40. K 0911 x K 0307 & $5.14 *$ & 62.93 & $-6.35^{*}$ & 45.87 & $0.33^{* *}$ & 2.72 & $-0.30 * *$ & 1.82 \\
\hline 41. K 0911 x NW 2036 & $11.05^{* *}$ & 64.33 & 2.98 & 53.20 & $0.27 * *$ & 2.43 & 0.14 & 2.24 \\
\hline 42. K 0911 x K 9423 & -1.01 & 52.40 & $12.09 * *$ & 60.47 & -0.08 & 2.06 & $0.43 * *$ & 2.34 \\
\hline 43. K 0307 x NW 2036 & -1.28 & 55.67 & $12.93^{* *}$ & 65.80 & $-0.23 * *$ & 2.21 & $0.25 * *$ & 2.51 \\
\hline 44. K 0307 x K 9423 & $8.32 * *$ & 65.40 & -1.55 & 49.47 & $0.51 * *$ & 2.92 & -0.11 & 1.96 \\
\hline 45. NW 2036 x K 9423 & -1.71 & 50.87 & $-6.43^{*}$ & 42.60 & -0.12 & 2.06 & $-0.27 * *$ & 1.78 \\
\hline $\mathrm{SE} \pm(\mathrm{Sij})$ & \multicolumn{2}{|l|}{2.40} & \multicolumn{2}{|l|}{2.49} & \multicolumn{2}{|l|}{0.074} & \multicolumn{2}{|l|}{0.088} \\
\hline $\mathrm{SE} \pm($ Sij-Sik) & \multicolumn{2}{|l|}{3.50} & \multicolumn{2}{|l|}{3.66} & \multicolumn{2}{|l|}{0.108} & \multicolumn{2}{|l|}{\begin{tabular}{|l|}
0.129 \\
\end{tabular}} \\
\hline
\end{tabular}


Int.J.Curr.Microbiol.App.Sci (2017) 6(3): 24-39

\begin{tabular}{|c|c|c|c|c|c|c|c|c|}
\hline \multirow[t]{3}{*}{ Crosses } & \multicolumn{4}{|c|}{ Spike length $(\mathrm{cm})$} & \multicolumn{4}{|c|}{ Biological yield per plant (g) } \\
\hline & \multicolumn{2}{|c|}{$\mathbf{F}_{1}$} & \multicolumn{2}{|c|}{$\mathbf{F}_{2}$} & \multicolumn{2}{|c|}{$F_{1}$} & \multicolumn{2}{|c|}{$\mathbf{F}_{2}$} \\
\hline & $\begin{array}{c}S C A \\
\text { effect }\end{array}$ & Mean & $\begin{array}{c}S C A \\
\text { effect }\end{array}$ & Mean & $\begin{array}{c}S C A \\
\text { effect }\end{array}$ & Mean & $\begin{array}{c}S C A \\
\text { effect }\end{array}$ & Mean \\
\hline 1.K 9533 x K 9162 & 0.12 & 11.83 & 0.04 & 11.60 & $1.54 * *$ & 24.71 & -0.06 & 20.94 \\
\hline $2 . \mathrm{K} 9533 \times \mathrm{K} 1114$ & $1.36^{* * *}$ & 12.67 & $1.01 * *$ & 11.97 & $3.54 * *$ & 26.97 & $-1.25^{*}$ & 18.53 \\
\hline 3. K $9533 \times$ DBW 14 & $-0.50 * *$ & 11.13 & $0.86^{* * *}$ & 12.23 & $-3.27 * *$ & 18.49 & $-3.09 * *$ & 16.72 \\
\hline $4 . \mathrm{K} 9533$ x K 0607 & 0.01 & 12.17 & $-1.21 * *$ & 10.37 & $4.48 * *$ & 28.21 & $3.23 * *$ & 23.53 \\
\hline $5 . \mathrm{K} 9533 \times \mathrm{K} 0424$ & $-0.56 * *$ & 11.23 & $0.58 * *$ & 12.00 & $-0.93 *$ & 20.77 & $-3.07 * *$ & 16.25 \\
\hline $6 . \mathrm{K} 9533 \times \mathrm{K} 0911$ & $0.28^{*}$ & 12.13 & $-0.91 * *$ & 10.50 & 0.46 & 24.62 & -1.01 & 19.90 \\
\hline 7. K $9533 \times$ K 0307 & $0.61 * *$ & 13.03 & 0.17 & 12.17 & $3.49 * *$ & 29.23 & $7.05 * *$ & 28.60 \\
\hline 8. K 9533 x NW 2036 & $0.34 * *$ & 12.13 & 0.23 & 11.70 & -0.64 & 21.78 & -1.17 & 19.08 \\
\hline 9. K 9533 x K 9423 & $-0.46 * *$ & 11.17 & $-0.72 * *$ & 10.37 & -0.48 & 21.99 & $3.33 * *$ & 24.30 \\
\hline 10. K 9162 x K 1114 & $-0.86^{* *}$ & 10.30 & 0.21 & 11.43 & $-2.45^{* *}$ & 20.69 & $-2.53^{* *}$ & 16.90 \\
\hline 11.K $9162 \times$ DBW 14 & -0.18 & 11.30 & 0.06 & 11.70 & $-2.70^{* *}$ & 18.76 & -0.88 & 18.58 \\
\hline 12. K 9162 × K 0607 & $0.32 * *$ & 12.33 & 0.16 & 12.00 & $-1.55 * *$ & 21.89 & 0.69 & 20.64 \\
\hline 13. K $9162 \times$ × 0424 & 0.15 & 11.80 & $-0.48 * *$ & 11.20 & $1.24 * *$ & 22.65 & 0.46 & 19.43 \\
\hline 14. K 9162 x K 0911 & $-0.48 * *$ & 11.23 & -0.14 & 11.53 & $-0.89 *$ & 22.98 & $1.94 * *$ & 22.50 \\
\hline 15. K9162 x K 0307 & $0.79 * *$ & 13.07 & $0.44 *$ & 12.70 & $2.99 * *$ & 28.43 & 0.53 & 21.73 \\
\hline 16. K 9162 x NW 2036 & $-1.02 * *$ & 10.63 & -0.77 ** & 10.97 & 0.76 & 22.89 & $-2.71 * *$ & 17.19 \\
\hline 17. K 9162 x K 9423 & $0.75^{\text {*** }}$ & 12.23 & $0.58 * *$ & 11.93 & $7.62 * *$ & 29.80 & $4.65^{* *}$ & 25.26 \\
\hline 18. K $1114 \times$ x DBW 14 & $0.96 * *$ & 12.03 & $0.50 * *$ & 11.53 & $-2.13 * *$ & 19.59 & -0.29 & 17.95 \\
\hline 19. K 1114 x K 0607 & $-1.13 * *$ & 10.47 & $0.76^{* * *}$ & 12.00 & $-8.04 * *$ & 15.65 & $-1.60 *$ & 17.12 \\
\hline 20. K 1114 x K 0424 & $1.13^{* * *}$ & 12.37 & $-0.81 * *$ & 10.27 & $6.80 * *$ & 28.46 & 0.29 & 18.05 \\
\hline 21. K 1114 x K 0911 & $-0.27 *$ & 11.03 & 0.03 & 11.10 & $4.59 * *$ & 28.72 & 0.89 & 20.23 \\
\hline 22. K 1114 x K 0307 & $-0.66 * *$ & 11.20 & $-0.79 * *$ & 10.87 & $-1.94 * *$ & 23.76 & $-1.56^{*}$ & 18.43 \\
\hline 23. K 1114 x NW 2036 & -0.04 & 11.20 & -0.10 & 11.03 & $7.71 * *$ & 30.10 & $3.56^{* * *}$ & 22.25 \\
\hline 24. K 1114 x K 9423 & $0.80 * *$ & 11.87 & 0.22 & 10.97 & $-2.71 * *$ & 19.73 & $-2.53 * *$ & 16.87 \\
\hline 25. DBW 14 x K 0607 & $0.84 * *$ & 12.77 & 0.28 & 11.93 & $2.61 * *$ & 24.63 & $2.30 * *$ & 21.06 \\
\hline 26. DBW 14 x K 0424 & 0.20 & 11.77 & 0.10 & 11.60 & $11.83^{* * *}$ & 31.81 & $4.90 * *$ & 22.69 \\
\hline 27. DBW 14 x K 0911 & $-0.66 * *$ & 10.97 & $-0.56 * *$ & 10.93 & $-1.43 * *$ & 21.02 & $-2.26 * *$ & 17.11 \\
\hline 28. DBW 14 x K 0307 & $0.48 * *$ & 12.67 & 0.02 & 12.10 & $-1.84 * *$ & 22.19 & $-3.68 * *$ & 16.34 \\
\hline 29. DBW 14 x NW 2036 & $-0.67 * *$ & 10.90 & $-0.48 * *$ & 11.07 & $-1.38^{* * *}$ & 19.33 & $3.06^{* * *}$ & 21.78 \\
\hline 30. DBW 14 x K 9423 & $0.33^{* * *}$ & 11.73 & 0.13 & 11.30 & 0.32 & 21.08 & $-1.62 *$ & 17.81 \\
\hline 31. K 0607 x K 0424 & -0.16 & 11.93 & 0.24 & 11.93 & $1.68 * *$ & 23.65 & $3.36 * *$ & 21.63 \\
\hline 32. K 0607 x K 0911 & $0.78^{* * *}$ & 12.93 & $0.38^{*}$ & 12.07 & $5.82 * *$ & 30.25 & 0.97 & 20.82 \\
\hline 33. K 0607 x K 0307 & -0.05 & 12.67 & $-0.68 * *$ & 11.60 & $2.32 * *$ & 28.32 & $-3.79 * *$ & 16.71 \\
\hline 34. K 0607 x NW 2036 & $0.38^{* * *}$ & 12.47 & 0.18 & 11.93 & $2.22 * *$ & 24.90 & $-4.61 * *$ & 14.59 \\
\hline 35. K 0607 x K 9423 & $0.44 * *$ & 12.37 & 0.13 & 11.50 & $-2.00 * *$ & 20.73 & $-2.52 * *$ & 17.40 \\
\hline 36. K 0424 x K 0911 & $-0.29 * *$ & 11.50 & $-0.50 * *$ & 11.03 & $-5.64 * *$ & 16.76 & $-2.15 * *$ & 16.74 \\
\hline 37. K 0424 x K 0307 & $-1.35 * *$ & 11.00 & $-0.75 * *$ & 11.37 & $-1.53 * *$ & 22.44 & -0.41 & 19.12 \\
\hline 38. K 0424 x NW 2036 & $-0.70 * *$ & 11.03 & $-0.36^{*}$ & 11.23 & $-2.86^{* * *}$ & 17.79 & 0.98 & 19.21 \\
\hline 39. K 0424 x K 9423 & $1.04 * *$ & 12.60 & $1.06^{* *}$ & 12.27 & $-2.80 * *$ & 17.91 & $-1.80 * *$ & 17.15 \\
\hline 40. K 0911 x K 0307 & $-0.62 * *$ & 11.80 & -0.14 & 11.97 & $4.13^{* * *}$ & 30.57 & $-2.94 * *$ & 18.18 \\
\hline 41. K 0911 x NW 2036 & $0.74 * *$ & 12.53 & 0.28 & 11.87 & $2.99 * *$ & 26.11 & $2.23^{* * *}$ & 22.05 \\
\hline 42. K 0911 x K 9423 & $0.34 * *$ & 11.97 & $0.73 * *$ & 11.93 & $1.44 * *$ & 24.60 & $5.03 * *$ & 25.56 \\
\hline 43. K 0307 x NW 2036 & -0.09 & 12.27 & -0.01 & 12.17 & -0.68 & 24.02 & $3.27 * *$ & 23.73 \\
\hline 44. K 0307 x K 9423 & -0.15 & 12.03 & 0.14 & 11.93 & $2.72 * *$ & 27.46 & $-1.30 *$ & 19.88 \\
\hline 45. NW 2036 x K 9423 & $-0.50 * *$ & 11.07 & $-0.70 * *$ & 10.57 & $-2.66^{* * *}$ & 18.77 & $-3.57 * *$ & 16.30 \\
\hline $\mathrm{SE} \pm(\mathrm{Sij})$ & 0.105 & & 0.164 & & 0.39 & & 0.60 & \\
\hline $\mathrm{SE} \pm($ Sij-Sik) & 0.154 & & 0.241 & & 0.58 & & 0.89 & \\
\hline
\end{tabular}


Int.J.Curr.Microbiol.App.Sci (2017) 6(3): 24-39

\begin{tabular}{|c|c|c|c|c|c|c|c|c|}
\hline \multirow[t]{3}{*}{ Crosses } & \multicolumn{4}{|c|}{ Harvest index (\%) } & \multicolumn{4}{|c|}{1000 grain weight $(\mathrm{g})$} \\
\hline & \multicolumn{2}{|c|}{$\mathbf{F}_{1}$} & \multicolumn{2}{|c|}{$\mathbf{F}_{2}$} & \multicolumn{2}{|c|}{$\mathbf{F}_{1}$} & \multicolumn{2}{|c|}{$\mathbf{F}_{2}$} \\
\hline & $\begin{array}{c}S C A \\
\text { effect }\end{array}$ & Mean & $\begin{array}{c}S C A \\
\text { effect }\end{array}$ & Mean & $\begin{array}{c}S C A \\
\text { effect }\end{array}$ & Mean & $\begin{array}{c}S C A \\
\text { effect }\end{array}$ & Mean \\
\hline 1.K 9533 x K 9162 & 0.91 & 45.85 & -2.72 & 40.34 & -0.23 & 39.90 & $-0.91 *$ & 39.58 \\
\hline 2.K $9533 \times$ K 1114 & $-3.32 * *$ & 41.01 & -1.74 & 44.07 & -0.23 & 40.18 & -0.12 & 39.78 \\
\hline 3. K $9533 \times$ DBW 14 & 1.48 & 46.36 & 1.70 & 45.81 & 0.25 & 39.26 & $1.55 * *$ & 41.44 \\
\hline 4.K 9533 x K 0607 & 0.80 & 46.49 & -1.99 & 42.07 & 0.02 & 40.28 & $-1.15^{* *}$ & 39.51 \\
\hline $5 . \mathrm{K} 9533 \times \mathrm{K} 0424$ & $-4.13 * *$ & 41.64 & 1.49 & 45.70 & $-1.60 * *$ & 38.28 & $-1.33^{* *}$ & 38.78 \\
\hline $6 . \mathrm{K} 9533 \times \mathrm{K} 0911$ & $3.58 * *$ & 48.80 & $5.61 * *$ & 51.04 & 0.56 & 40.73 & 1.89 ** & 42.23 \\
\hline 7. K $9533 \times$ K 0307 & $6.46^{* *}$ & 54.06 & -3.24 & 41.55 & $1.69^{* *}$ & 43.35 & $0.85 *$ & 41.95 \\
\hline 8. K 9533 x NW 2036 & $-5.60 * *$ & 37.96 & -1.75 & 41.99 & $-1.61 * *$ & 39.17 & -0.55 & 40.84 \\
\hline 9. K 9533 x K 9423 & 0.30 & 46.97 & -0.92 & 42.17 & -0.28 & 39.68 & $-0.95 *$ & 39.00 \\
\hline 10. K 9162 x K 1114 & $-4.49 * *$ & 41.31 & -3.06 & 44.34 & $-1.41^{* * *}$ & 40.33 & 0.12 & 41.34 \\
\hline 11.K $9162 \times$ DBW 14 & $-4.14 * *$ & 42.20 & -2.24 & 43.47 & $-1.44 * *$ & 38.90 & $-0.77 *$ & 40.44 \\
\hline 12. K 9162 × K 0607 & $3.25 * *$ & 50.40 & 3.29 & 48.94 & $2.46^{* * *}$ & 44.05 & $2.10 * *$ & 44.09 \\
\hline 13. K $9162 \times$ K 0424 & $5.16^{* * *}$ & 52.40 & -0.17 & 45.63 & $-1.10^{* * *}$ & 40.11 & -0.43 & 41.00 \\
\hline 14. K 9162 x K 0911 & -0.58 & 46.11 & $-6.87 * *$ & 40.16 & -0.01 & 41.49 & 0.29 & 41.95 \\
\hline 15. K9162 x K 0307 & $2.38^{*}$ & 51.45 & -2.50 & 43.89 & $1.30 * *$ & 44.29 & 0.28 & 42.69 \\
\hline 16. K 9162 x NW 2036 & $-20.04 * *$ & 24.99 & 2.49 & 47.82 & -0.61 & 41.50 & $-1.05^{* *}$ & 41.66 \\
\hline 17. K 9162 x K 9423 & 7.20 ** & 55.33 & -2.08 & 42.61 & $2.69 * *$ & 43.98 & $2.72 * *$ & 44.00 \\
\hline 18. K $1114 \times$ x DBW 14 & -0.16 & 45.58 & $-6.20 * *$ & 42.27 & -0.37 & 40.26 & -0.49 & 40.13 \\
\hline 19. K $1114 \times$ × K 0607 & $-4.08 * *$ & 42.47 & -2.61 & 45.80 & $-2.31 * *$ & 39.56 & 0.72 & 42.11 \\
\hline 20. K 1114 x K 0424 & $8.83^{* * *}$ & 55.46 & -2.82 & 45.75 & $1.16^{* * *}$ & 42.65 & $-3.89 * *$ & 36.95 \\
\hline 21. K 1114 x K 0911 & 1.23 & 47.31 & 2.09 & 51.88 & $-1.45^{* *}$ & 40.33 & $-1.14 * *$ & 39.94 \\
\hline 22. K 1114 x K 0307 & $-6.20 * *$ & 42.26 & 0.64 & 49.78 & $0.79 *$ & 44.06 & 0.03 & 41.86 \\
\hline 23. K 1114 x NW 2036 & $7.17 * *$ & 51.58 & -2.26 & 45.84 & $3.52 * *$ & 45.90 & $2.00 * *$ & 44.12 \\
\hline 24. K 1114 x K 9423 & $-14.69 * *$ & 32.83 & $10.22 * *$ & 57.67 & $-1.31 * *$ & 40.26 & $-1.66 * *$ & 39.03 \\
\hline 25. DBW 14 x K 0607 & $2.81 *$ & 49.90 & 2.32 & 49.03 & $-1.35^{* * *}$ & 39.12 & $-0.85^{*}$ & 40.54 \\
\hline 26. DBW 14 x K 0424 & $6.39 * *$ & 53.57 & 2.64 & 49.51 & $4.31 * *$ & 44.40 & $2.12 * *$ & 42.96 \\
\hline 27. DBW 14 x K 0911 & $-9.34 * *$ & 37.29 & $9.29 * *$ & 57.38 & $2.03 * *$ & 42.40 & $-1.85^{* *}$ & 39.22 \\
\hline 28. DBW 14 x K 0307 & -1.75 & 47.25 & 2.97 & 50.41 & $-4.20 * *$ & 37.67 & $1.34 * *$ & 43.16 \\
\hline 29. DBW 14 x NW 2036 & 1.80 & 46.76 & 0.49 & 46.89 & $1.88^{* * *}$ & 42.87 & $1.05 * *$ & 43.17 \\
\hline 30. DBW 14 x K 9423 & 8.67 ** & 56.74 & $-4.23 *$ & 41.52 & $-1.54 * *$ & 38.63 & 0.25 & 40.93 \\
\hline 31. K 0607 x K 0424 & $-2.47 *$ & 45.51 & 1.12 & 47.92 & 0.15 & 41.48 & $1.03 * *$ & 42.64 \\
\hline 32. K 0607 x K 0911 & -0.79 & 46.64 & $-4.42 *$ & 43.61 & $3.30 * *$ & 44.92 & $1.68 * *$ & 43.52 \\
\hline 33. K 0607 x K 0307 & $-3.77 * *$ & 46.05 & -0.45 & 46.93 & $0.98 * *$ & 44.09 & $-2.27 * *$ & 40.32 \\
\hline 34. K 0607 x NW 2036 & $2.18^{*}$ & 47.95 & 3.05 & 49.39 & $-5.14 * *$ & 37.09 & $-2.62 * *$ & 40.27 \\
\hline 35. K 0607 x K 9423 & -0.62 & 48.26 & $-5.50 * *$ & 40.19 & $-1.31 * *$ & 40.11 & $-0.94 *$ & 40.51 \\
\hline 36. K 0424 x K 0911 & $-9.06 * *$ & 38.46 & -0.04 & 48.15 & $-3.44 * *$ & 37.80 & $-1.43 * *$ & 39.86 \\
\hline 37. K 0424 x K 0307 & $-3.64 * *$ & 46.26 & -1.78 & 45.76 & $-1.28 * *$ & 41.45 & $2.54 * *$ & 44.58 \\
\hline 38. K 0424 x NW 2036 & 1.62 & 47.48 & 0.77 & 47.26 & $3.55^{* * *}$ & 45.40 & $3.41 * *$ & 45.74 \\
\hline 39. K 0424 x K 9423 & 0.81 & 49.77 & 0.09 & 45.94 & $2.41 * *$ & 43.44 & $2.35 * *$ & 43.25 \\
\hline 40. K 0911 x K 0307 & -0.42 & 48.94 & $-6.93 * *$ & 41.83 & $1.06^{* *}$ & 44.08 & $-1.38 * *$ & 40.90 \\
\hline 41. K 0911 x NW 2036 & $4.25 * *$ & 49.56 & -3.53 & 44.19 & -0.02 & 42.11 & $1.05^{* * *}$ & 43.62 \\
\hline 42. K 0911 x K 9423 & -0.49 & 47.92 & -1.96 & 45.11 & $-1.12 * *$ & 40.21 & $1.79 * *$ & 42.93 \\
\hline 43. K 0307 x NW 2036 & $3.07 * *$ & 50.75 & 2.43 & 49.50 & $-2.33 * *$ & 41.30 & $-1.97 * *$ & 41.36 \\
\hline 44. K 0307 x K 9423 & $2.23^{*}$ & 53.02 & 0.01 & 46.44 & $2.23 * *$ & 45.05 & $-2.17 * *$ & 39.72 \\
\hline 45. NW 2036 x K 9423 & $6.22^{* *}$ & 52.97 & $3.73^{*}$ & 49.11 & 0.14 & 42.07 & -0.21 & 41.98 \\
\hline $\mathrm{SE} \pm(\mathrm{Sij})$ & 1.06 & & 1.80 & & 0.32 & & 0.38 & \\
\hline $\mathrm{SE} \pm($ Sij-Sik) & 1.57 & & 2.65 & & 0.47 & & 0.56 & \\
\hline
\end{tabular}


Int.J.Curr.Microbiol.App.Sci (2017) 6(3): 24-39

\begin{tabular}{|c|c|c|c|c|c|c|c|c|}
\hline \multirow[t]{3}{*}{ Crosses } & \multicolumn{4}{|c|}{ Spike density } & \multicolumn{4}{|c|}{ Protein content (\%) } \\
\hline & \multicolumn{2}{|c|}{$\mathrm{F}_{1}$} & \multicolumn{2}{|c|}{$\mathbf{F}_{2}$} & \multicolumn{2}{|c|}{$\mathbf{F}_{1}$} & \multicolumn{2}{|c|}{$\mathbf{F}_{2}$} \\
\hline & $\begin{array}{c}S C A \\
\text { effect }\end{array}$ & Mean & $\begin{array}{c}S C A \\
\text { effect }\end{array}$ & Mean & $\begin{array}{c}S C A \\
\text { effect }\end{array}$ & Mean & $\begin{array}{c}S C A \\
\text { effect }\end{array}$ & Mean \\
\hline $1 . \mathrm{K} 9533 \times \mathrm{K} 9162$ & $0.11^{*}$ & 1.77 & 0.01 & 1.70 & $1.10^{* *}$ & 11.77 & $0.75^{* *}$ & 11.60 \\
\hline 2.K $9533 \times$ K 1114 & 0.03 & 1.74 & -0.02 & 1.75 & $1.12 * *$ & 12.00 & $1.28 * *$ & 12.13 \\
\hline 3. K $9533 \times$ DBW 14 & 0.02 & 1.77 & $-0.19 * *$ & 1.57 & $-0.40 * *$ & 10.63 & $0.36^{*}$ & 11.20 \\
\hline 4.K 9533 x K 0607 & 0.05 & 1.69 & $0.21 * *$ & 1.92 & $-1.19 * *$ & 10.17 & -0.66 ** & 10.57 \\
\hline $5 . \mathrm{K} 9533 \times \mathrm{K} 0424$ & $-0.25 * *$ & 1.39 & -0.07 & 1.64 & $-1.30^{* *}$ & 10.27 & $-0.85^{* *}$ & 10.27 \\
\hline $6 . K 9533 \times$ K 0911 & 0.06 & 1.75 & $0.20 * *$ & 1.95 & $-0.84 * *$ & 10.83 & $-1.28 * *$ & 10.03 \\
\hline 7. K $9533 \times$ K 0307 & -0.03 & 1.61 & -0.02 & 1.65 & $-0.59^{* *}$ & 10.70 & $-1.27 * *$ & 9.97 \\
\hline 8. K 9533 x NW 2036 & -0.06 & 1.64 & $-0.08 *$ & 1.66 & $-1.02 * *$ & 10.27 & 0.00 & 11.13 \\
\hline 9. K $9533 \times$ K 9423 & 0.04 & 1.76 & $0.08^{*}$ & 1.85 & -0.20 & 11.21 & $-1.01 * *$ & 9.87 \\
\hline 10. K 9162 x K 1114 & 0.02 & 1.71 & $-0.08 *$ & 1.61 & $-0.47^{* *}$ & 11.37 & 0.00 & 11.67 \\
\hline 11.K $9162 \times$ DBW 14 & $-0.22 * *$ & 1.50 & $-0.20 * *$ & 1.49 & $-0.66^{* *}$ & 11.33 & 0.18 & 11.83 \\
\hline 12. K 9162 × K 0607 & -0.02 & 1.61 & 0.01 & 1.64 & $-0.62 * *$ & 11.70 & $-1.08 * *$ & 10.97 \\
\hline 13. K $9162 \times$ K 0424 & 0.05 & 1.68 & $0.08^{*}$ & 1.71 & $-0.99 * *$ & 11.53 & $0.74 * *$ & 12.67 \\
\hline 14. K 9162 x K 0911 & 0.02 & 1.70 & 0.01 & 1.68 & $-0.64 * *$ & 12.00 & $-1.29 * *$ & 10.83 \\
\hline 15. K9162 x K 0307 & $-0.10^{*}$ & 1.53 & $-0.09 *$ & 1.50 & 0.02 & 12.27 & $-0.52 * *$ & 11.53 \\
\hline 16. K 9162 x NW 2036 & 0.08 & 1.77 & $0.13 * *$ & 1.78 & $0.75 * *$ & 13.00 & -0.28 & 11.67 \\
\hline 17. K 9162 x K 9423 & -0.01 & 1.69 & -0.04 & 1.64 & $-1.13^{* *}$ & 11.23 & $-1.10^{* * *}$ & 10.60 \\
\hline 18. K $1114 \times$ x DBW 14 & $-0.14 * *$ & 1.62 & 0.00 & 1.76 & $-0.44 * *$ & 11.77 & -0.32 & 11.33 \\
\hline 19. K 1114 x K 0607 & $-0.15 * *$ & 1.51 & 0.04 & 1.75 & $-2.26 * *$ & 10.27 & $-0.75^{* * *}$ & 11.30 \\
\hline 20. K 1114 x K 0424 & $0.14 * *$ & 1.81 & -0.04 & 1.67 & $-0.30 * *$ & 12.43 & $-0.50 * *$ & 11.43 \\
\hline 21. K 1114 x K 0911 & $0.09 *$ & 1.81 & 0.03 & 1.79 & $0.98^{* * *}$ & 13.83 & $1.54 * *$ & 13.67 \\
\hline 22. K 1114 x K 0307 & 0.01 & 1.68 & $0.12 * *$ & 1.79 & $0.77 * *$ & 13.23 & $-0.42 *$ & 11.63 \\
\hline 23. K 1114 x NW 2036 & 0.00 & 1.72 & -0.01 & 1.72 & $1.18^{* *}$ & 13.63 & 0.09 & 12.03 \\
\hline 24. K 1114 x K 9423 & $-0.12 * *$ & 1.62 & $-0.10^{* *}$ & 1.66 & $1.02 * *$ & 13.60 & -0.10 & 11.60 \\
\hline 25. DBW 14 x K 0607 & 0.03 & 1.73 & -0.03 & 1.67 & $1.34 * *$ & 14.03 & $0.53 * *$ & 12.57 \\
\hline 26. DBW 14 x K 0424 & 0.17 ** & 1.88 & $0.09 *$ & 1.79 & $1.54 * *$ & 14.43 & 0.22 & 12.13 \\
\hline 27. DBW 14 x K 0911 & 0.05 & 1.80 & 0.02 & 1.78 & $0.66 * *$ & 13.67 & $1.09 * *$ & 13.20 \\
\hline 28. DBW 14 x K 0307 & -0.05 & 1.66 & 0.06 & 1.73 & $-0.69 * *$ & 11.93 & $-0.38 *$ & 11.67 \\
\hline 29. DBW 14 x NW 2036 & 0.17 ** & 1.93 & $0.16^{* * *}$ & 1.89 & $1.15^{* * *}$ & 13.77 & $0.90 * *$ & 12.83 \\
\hline 30. DBW 14 x K 9423 & -0.04 & 1.74 & -0.01 & 1.75 & $1.66^{* *}$ & 14.40 & 0.11 & 11.80 \\
\hline 31. K 0607 x K 0424 & $0.09 *$ & 1.69 & 0.11 ** & 1.76 & 0.01 & 13.23 & 0.19 & 12.50 \\
\hline 32. K 0607 x K 0911 & 0.08 & 1.73 & $-0.08 *$ & 1.62 & $1.73^{* *} *$ & 15.06 & -0.01 & 12.50 \\
\hline 33. K 0607 x K 0307 & 0.01 & 1.61 & 0.00 & 1.61 & $1.94 * *$ & 14.89 & $1.93 * *$ & 14.37 \\
\hline 34. K 0607 x NW 2036 & $-0.18 * *$ & 1.48 & $-0.27 * *$ & 1.40 & $0.53 * *$ & 13.47 & $0.41 *$ & 12.73 \\
\hline 35. K 0607 x K 9423 & -0.03 & 1.65 & -0.03 & 1.68 & $0.89 * *$ & 13.95 & $0.65 * *$ & 12.73 \\
\hline 36. K 0424 x K 0911 & $-0.21 * *$ & 1.44 & 0.07 & 1.77 & $0.67 * *$ & 14.21 & -0.02 & 12.37 \\
\hline 37. K 0424 x K 0307 & $0.12 * *$ & 1.73 & 0.05 & 1.66 & $0.79 * *$ & 13.94 & $-0.82 * *$ & 11.50 \\
\hline 38. K 0424 x NW 2036 & 0.06 & 1.72 & 0.02 & 1.69 & $0.43 * *$ & 13.57 & 0.19 & 12.40 \\
\hline 39. K 0424 x K 9423 & -0.03 & 1.65 & $-0.09 *$ & 1.61 & $1.30 * *$ & 14.56 & $0.54 * *$ & 12.50 \\
\hline 40. K $0911 \times$ K 0307 & $0.13^{* *}$ & 1.78 & 0.00 & 1.67 & 0.02 & 13.28 & $0.95 * *$ & 13.47 \\
\hline 41. K 0911 x NW 2036 & 0.02 & 1.73 & 0.01 & 1.74 & $0.38 * *$ & 13.63 & -0.01 & 12.40 \\
\hline 42. K 0911 x K 9423 & $-0.14 * *$ & 1.59 & $-0.07 *$ & 1.68 & $0.48^{* * *}$ & 13.86 & 0.34 & 12.50 \\
\hline 43. K 0307 x NW 2036 & 0.04 & 1.70 & 0.05 & 1.69 & $-0.49^{* *}$ & 12.38 & -0.04 & 12.30 \\
\hline 44. K 0307 x K 9423 & 0.05 & 1.73 & -0.04 & 1.63 & $-1.13^{* *}$ & 11.86 & 0.35 & 12.43 \\
\hline 45. NW 2036 x K 9423 & -0.06 & 1.67 & 0.00 & 1.73 & $0.43^{\text {** }}$ & 13.41 & $0.79 * *$ & 12.77 \\
\hline $\mathrm{SE} \pm(\mathrm{Sij})$ & \multicolumn{2}{|l|}{0.042} & \multicolumn{2}{|l|}{0.035} & \multicolumn{2}{|l|}{0.10} & \multicolumn{2}{|l|}{0.17} \\
\hline $\mathrm{SE} \pm($ Sij-Sik) & \multicolumn{2}{|l|}{0.061} & \multicolumn{2}{|l|}{0.051} & \multicolumn{2}{|l|}{0.15} & \multicolumn{2}{|l|}{0.25} \\
\hline
\end{tabular}




\begin{tabular}{|c|c|c|c|c|}
\hline \multirow[t]{3}{*}{ Crosses } & \multicolumn{4}{|c|}{ Grain yield per plant (g) } \\
\hline & \multicolumn{2}{|c|}{$\mathbf{F}_{1}$} & \multicolumn{2}{|l|}{$\mathbf{F}_{2}$} \\
\hline & $\begin{array}{c}S C A \\
\text { effect }\end{array}$ & Mean & $\begin{array}{c}S C A \\
\text { effect }\end{array}$ & Mean \\
\hline $1 . \mathrm{K} 9533 \times \mathrm{K} 9162$ & $0.85 *$ & 11.33 & -0.55 & 8.44 \\
\hline 2.K $9533 \times$ X K 1114 & 0.56 & 11.05 & $-0.85^{*}$ & 8.16 \\
\hline 3. K $9533 \times$ x DBW 14 & $-1.26^{* * *}$ & 8.57 & $-1.03^{*}$ & 7.65 \\
\hline $4 . K 9533 \times$ K 0607 & $2.26^{* *}$ & 13.12 & $0.97 *$ & 9.86 \\
\hline $5 . \mathrm{K} 9533 \times \mathrm{K} 0424$ & $-1.41 * *$ & 8.64 & $-1.10^{* *}$ & 7.38 \\
\hline $6 . \mathrm{K} 9533 \times \mathrm{K} 0911$ & $1.05^{* *}$ & 12.04 & 0.70 & 10.11 \\
\hline 7. K $9533 \times 1 \mathrm{~K} 0307$ & $3.48^{* *}$ & 15.80 & $2.25 * *$ & 11.88 \\
\hline 8. K $9533 \times$ xW 2036 & $-1.53^{* *}$ & 8.27 & -0.79 & 8.03 \\
\hline 9. K $9533 \times$ K K 9423 & -0.21 & 10.33 & $1.28^{* *}$ & 10.26 \\
\hline 10. K 9162 x K 1114 & $-2.15^{* *}$ & 8.55 & $-1.68^{* *}$ & 7.51 \\
\hline 11.K $9162 \times$ DBW 14 & $-2.11 * *$ & 7.92 & $-0.85^{*}$ & 8.01 \\
\hline 12. K $9162 \times$ K 0607 & -0.03 & 11.03 & $0.95 *$ & 10.02 \\
\hline 13. K $9162 \times$ K 0424 & $1.63 * *$ & 11.88 & 0.17 & 8.84 \\
\hline 14. K 9162 x K 0911 & -0.61 & 10.59 & -0.54 & 9.04 \\
\hline 15. K9162 x K 0307 & $2.11^{* *}$ & 14.63 & -0.28 & 9.53 \\
\hline 16. K 9162 x NW 2036 & $-4.29 * *$ & 5.71 & -0.71 & 8.29 \\
\hline 17. K 9162 × K 9423 & $5.73^{* *}$ & 16.49 & $1.61^{* *}$ & 10.77 \\
\hline 18. K $1114 \times$ x DBW 14 & $-1.14 * *$ & 8.90 & $-1.29 * *$ & 7.59 \\
\hline 19. K $1114 \times$ x K 0607 & $-4.42 * *$ & 6.65 & $-1.23 * *$ & 7.86 \\
\hline 20. K 1114 x K 0424 & $5.52^{* *}$ & 15.78 & -0.45 & 8.24 \\
\hline 21. K 1114 x K 0911 & $2.38^{* *}$ & 13.59 & $0.89^{*}$ & 10.50 \\
\hline 22. K 1114 x K 0307 & $-2.50^{* * *}$ & 10.05 & -0.64 & 9.19 \\
\hline 23. K 1114 x NW 2036 & $5.52^{* *}$ & 15.53 & $1.16^{* * *}$ & 10.18 \\
\hline 24. K 1114 x K 9423 & $-4.29 * *$ & 6.47 & 0.56 & 9.73 \\
\hline 25. DBW 14 x K 0607 & $1.88^{* * *}$ & 12.29 & $1.55^{* *} *$ & 10.31 \\
\hline 26. DBW $14 \times$ K 0424 & $7.44^{* *}$ & 17.04 & $2.86^{* * *}$ & 11.21 \\
\hline 27. DBW 14 x K 0911 & $-2.70 * *$ & 7.84 & 0.54 & 9.82 \\
\hline 28. DBW 14 x K 0307 & $-1.39 * *$ & 10.49 & $-1.27 * *$ & 8.23 \\
\hline 29. DBW $14 \times$ NW 2036 & -0.33 & 9.02 & $1.53^{* *}$ & 10.22 \\
\hline 30. DBW 14 x K 9423 & $1.86^{* * *}$ & 11.96 & $-1.45^{* *}$ & 7.39 \\
\hline 31. K 0607 x K 0424 & 0.14 & 10.76 & $1.80^{* * *}$ & 10.37 \\
\hline 32. K 0607 x K 0911 & $2.54 * *$ & 14.11 & -0.40 & 9.08 \\
\hline 33. K 0607 x K 0307 & 0.13 & 13.03 & $-1.86^{* * *}$ & 7.85 \\
\hline 34. K 0607 x NW 2036 & $1.57 * *$ & 11.95 & $-1.71 * *$ & 7.19 \\
\hline 35. K 0607 x K 9423 & $-1.13^{* *}$ & 10.00 & $-2.05^{* *}$ & 7.00 \\
\hline 36. K 0424 x K 0911 & $-4.30 * *$ & 6.46 & $-1.02^{*}$ & 8.06 \\
\hline 37. K $0424 \times$ X 0307 & $-1.68 * *$ & 10.41 & -0.56 & 8.75 \\
\hline 38. K 0424 x NW 2036 & $-1.12 * *$ & 8.45 & 0.59 & 9.08 \\
\hline 39. K 0424 x K 9423 & $-1.40 * *$ & 8.92 & -0.78 & 7.87 \\
\hline 40. K 0911 x K 0307 & $1.90^{* *}$ & 14.94 & $-2.62 * *$ & 7.60 \\
\hline 41. K 0911 x NW 2036 & $2.42 * *$ & 12.94 & 0.33 & 9.74 \\
\hline 42. K 0911 x K 9423 & 0.56 & 11.82 & $1.96^{* * *}$ & 11.53 \\
\hline 43. K 0307 x NW 2036 & 0.36 & 12.21 & $2.10 * *$ & 11.74 \\
\hline 44. K 0307 x K 9423 & $1.96^{* * *}$ & 14.56 & -0.58 & 9.21 \\
\hline 45. NW 2036 x K 9423 & -0.13 & 9.94 & $-0.98 *$ & 8.01 \\
\hline $\mathrm{SE} \pm(\mathrm{Sij})$ & 0.32 & & 0.39 & \\
\hline SE $\pm($ Sij-Sik $)$ & 0.47 & & 0.57 & \\
\hline
\end{tabular}


Table.4 Ranking of the desirable parents on the basis of per se performance and gca effects for 11 th characters in bread wheat

\begin{tabular}{|c|c|c|c|c|c|}
\hline \multirow[t]{2}{*}{ Character } & \multirow{2}{*}{$\begin{array}{l}\text { Desirable parent } \\
\text { on the basis of per } \\
s e \text { performance }\end{array}$} & \multicolumn{2}{|c|}{ Good general combiner } & \multirow{2}{*}{$\begin{array}{l}\text { Common } \\
\text { parent in } F_{1} \text { and } \\
F_{2}\end{array}$} & \multirow{2}{*}{$\begin{array}{l}\text { Common parent on the } \\
\text { basis of per se and gca } \\
\text { effect in } F_{1} \text { and } F_{2}\end{array}$} \\
\hline & & $\mathrm{F}_{1}$ & $\mathrm{~F}_{2}$ & & \\
\hline $\begin{array}{l}\text { Number of } \\
\text { effective tillers per } \\
\text { plant }\end{array}$ & $\begin{array}{l}\text { K } 1114 \\
\text { K } 9162 \\
\text { K } 9423 \\
\text { K } 0307 \\
\text { K } 0911 \\
\end{array}$ & $\begin{array}{l}\text { K 0307** } \\
\text { DBW } 14 * *\end{array}$ & $\begin{array}{l}\text { K } 1114 * * \\
\text { K 0424** } \\
\text { K 0911** } \\
\text { K } 9162\end{array}$ & Nil & Nil \\
\hline $\begin{array}{l}\text { Number of } \\
\text { spikelets per spike }\end{array}$ & $\begin{array}{l}\text { NW } 2036 \\
\text { K } 0307 \\
\text { DBW } 14 \\
\text { K } 9162 \\
\text { K } 0607\end{array}$ & $\begin{array}{l}\text { K } 0307 * * \\
\text { K } 0607 * *\end{array}$ & $\begin{array}{l}\text { DBW } 14 * * \\
\text { K 0911** } \\
\text { K 0307** } \\
\text { K } 9533^{*}\end{array}$ & K 0307 & K 0307 \\
\hline $\begin{array}{l}\text { Number of grains } \\
\text { per spike }\end{array}$ & $\begin{array}{l}\text { K } 0307 \\
\text { NW } 2036 \\
\text { K } 0911 \\
\text { K } 0607 \\
\text { DBW } 14\end{array}$ & $\mathrm{~K} 0307 * *$ & K 0307** & K 0307 & K 0307 \\
\hline $\begin{array}{l}\text { Grains weight per } \\
\text { spike (g) }\end{array}$ & $\begin{array}{l}\text { K } 0307 \\
\text { NW } 2036 \\
\text { K } 0607 \\
\text { K } 0911 \\
\text { K } 1114 \\
\end{array}$ & $\begin{array}{l}\text { K } 1114 * * \\
\text { K } 0911 * * \\
\text { K } 0307 * *\end{array}$ & $\begin{array}{l}\text { K 0307*** } \\
\text { NW 2036** }\end{array}$ & K 0307 & K 0307 \\
\hline Spike length (cm) & $\begin{array}{l}\text { K } 0307 \\
\text { NW } 2036 \\
\text { K } 0424 \\
\text { K } 9162 \\
\text { K } 0607\end{array}$ & $\begin{array}{l}\text { K } 0307 * * \\
\text { K } 0911 * * \\
\text { K } 0607 * * \\
\text { K } 9533 * *\end{array}$ & $\begin{array}{l}\mathrm{K} 0307 * * \\
\mathrm{~K} 0607 * * \\
\mathrm{~K} 9162 * *\end{array}$ & $\begin{array}{l}\text { K } 0307 \\
\text { K } 0607\end{array}$ & $\begin{array}{l}\text { K } 0307 \\
\text { K } 0607\end{array}$ \\
\hline $\begin{array}{l}\text { Biological yield per } \\
\text { plant (g) }\end{array}$ & $\begin{array}{l}\text { K } 0307 \\
\text { K } 9423 \\
\text { K } 1114 \\
\text { K } 0607 \\
\text { K } 9162\end{array}$ & $\begin{array}{l}\text { K } 0307 * * \\
\text { K } 9423 * *\end{array}$ & $\begin{array}{l}\text { K 9533** } \\
\text { K 9162** } \\
\text { K 0911** } \\
\text { K 0307** } \\
\text { K 9423** }\end{array}$ & $\begin{array}{l}\text { K } 0307 \\
\text { K } 9423\end{array}$ & $\begin{array}{l}\text { K } 307 \\
\text { K } 9423\end{array}$ \\
\hline Harvest index (\%) & $\begin{array}{l}\text { K } 1114 \\
\text { K } 0911 \\
\text { K } 0307 \\
\text { K } 9162 \\
\text { K0607 } \\
\end{array}$ & $\begin{array}{l}\text { K 0307** } \\
\text { NW 2036** } \\
\text { K 1114** }\end{array}$ & $\begin{array}{l}\text { K 1114** } \\
\text { K 0911* }\end{array}$ & K 1114 & K 1114 \\
\hline $\begin{array}{l}\text { 1000 grain weight } \\
\text { (g) }\end{array}$ & $\begin{array}{l}\text { K } 0307 \\
\text { K } 0607 \\
\text { NW } 2036 \\
\text { K } 1114 \\
\text { K } 0911\end{array}$ & $\begin{array}{l}\text { K } 0307 * * \\
\text { K } 0607 * *\end{array}$ & $\begin{array}{l}\text { NW 2036** } \\
\text { K 0307** } \\
\text { DBW 14** } \\
\text { K 0607** }\end{array}$ & $\begin{array}{l}\text { K } 0307 \\
\text { K } 0607\end{array}$ & $\begin{array}{l}\text { K } 0307 \\
\text { K } 0607\end{array}$ \\
\hline Spike density & $\begin{array}{l}\text { K } 9423 \\
\text { DBW } 14 \\
\text { K } 1114 \\
\text { K } 9533 \\
\text { NW } 2036 \\
\end{array}$ & $\begin{array}{l}\text { DBW 14*** } \\
\text { K 9423** }\end{array}$ & $\begin{array}{l}\text { K 9533** } \\
\text { K 1114** } \\
\text { DBW 14** } \\
\text { K 9423** } \\
\text { K 0911* }\end{array}$ & $\begin{array}{l}\text { DBW } 14 \\
\text { K } 9423\end{array}$ & $\begin{array}{l}\text { DBW } 14 \\
\text { K } 9423\end{array}$ \\
\hline $\begin{array}{l}\text { Protein content } \\
(\%)\end{array}$ & $\begin{array}{l}\text { K } 9162 \\
\text { K } 0307 \\
\text { K } 0424 \\
\text { K } 0911 \\
\text { K } 9423 \\
\end{array}$ & $\begin{array}{l}\text { K 0911** } \\
\text { K 0424** } \\
\text { K 9423** } \\
\text { K 0607** } \\
\text { NW 2036** }\end{array}$ & $\begin{array}{l}\text { K } 9533^{* *} \\
\text { K } 0911^{* *} \\
\text { K 0307** } \\
\text { K 0607** } \\
\text { NW 2036** }\end{array}$ & $\begin{array}{l}\text { K } 0911 \\
\text { K } 0607 \\
\text { NW } 2036\end{array}$ & K 0911 \\
\hline $\begin{array}{l}\text { Grain yield per } \\
\text { plant }(\mathrm{g})\end{array}$ & $\begin{array}{l}\text { K } 0307 \\
\text { K } 1114 \\
\text { K } 9162 \\
\text { K } 0911 \\
\text { K } 0607 \\
\end{array}$ & $\begin{array}{l}\text { K 0307** } \\
\text { K 0911** } \\
\text { K 0607** }\end{array}$ & $\begin{array}{l}\text { K 0307** } \\
\text { K 0911** }\end{array}$ & $\begin{array}{l}\text { K } 0307 \\
\text { K } 0911\end{array}$ & $\begin{array}{l}\text { K } 0307 \\
\text { K } 0911\end{array}$ \\
\hline
\end{tabular}

*significant at 5\% and ** significant at $1 \%$. 
Table.5 Ranking of the crosses in respect to their superiority for specific combining ability, per se performance and general combining effects of the parents for 11th characters in bread wheat

\begin{tabular}{|c|c|c|c|}
\hline Character & Good specific combiner & $\begin{array}{l}\text { gca effect of } \\
\text { parent }\end{array}$ & $\begin{array}{l}\text { Superior crosses on the basis of } \\
\text { per se performance }\end{array}$ \\
\hline $\begin{array}{l}F_{1} s \\
\text { Number of effective tillers per } \\
\text { plant }\end{array}$ & $\begin{array}{l}\text { K } 1114 \text { x K 0911*** } \\
\text { K } 1114 \text { x NW 2036** } \\
\text { DBW } 14 \text { x K 0424*** } \\
\text { K } 9162 \text { x K 9423** } \\
\text { DBW } 14 \text { x K } 0607 * *\end{array}$ & $\begin{array}{l}\mathrm{L} \times \mathrm{A} \\
\mathrm{L} \times \mathrm{A} \\
\mathrm{H} \times \mathrm{L} \\
\mathrm{L} \times \mathrm{A} \\
\mathrm{H} \times \mathrm{A}\end{array}$ & $\begin{array}{l}\text { K } 1114 \text { x K } 0911 \\
\text { K } 1114 \text { x NW } 2036 \\
\text { K } 9162 \text { x K } 9423 \\
\text { DBW } 14 \text { x K } 0424 \\
\text { K } 9533 \text { x K } 0307 \\
\end{array}$ \\
\hline Number of spikelets per spike & $\begin{array}{l}\text { K } 1114 \text { x K 0424*** } \\
\text { K } 9533 \text { x K 1114*** } \\
\text { DBW } 14 \text { x K 0424** } \\
\text { K } 0607 \text { x K 0911*** } \\
\text { DBW } 14 \text { x K 0607** }\end{array}$ & $\begin{array}{l}\mathrm{L} \times \mathrm{L} \\
\mathrm{A} \times \mathrm{L} \\
\mathrm{A} \times \mathrm{L} \\
\mathrm{H} \times \mathrm{A} \\
\mathrm{A} \times \mathrm{H}\end{array}$ & $\begin{array}{l}\text { K } 1114 \text { x K } 0424 \\
\text { K } 0607 \text { x K } 0911 \\
\text { DBW } 14 \text { x K } 0607 \\
\text { DBW } 14 \text { x K } 0424 \\
\text { K } 9533 \text { × K } 1114 \\
\end{array}$ \\
\hline Number of grains per spike & $\begin{array}{l}\text { DBW } 14 \text { x K 0424*** } \\
\text { K } 1114 \text { x K 0607** } \\
\text { K } 9162 \text { x K 9423** } \\
\text { K } 0911 \text { x NW } 2036^{* *} \\
\text { K 0607 x K 0911** } \\
\end{array}$ & $\begin{array}{l}\mathrm{L} \times \mathrm{A} \\
\mathrm{L} \times \mathrm{A} \\
\mathrm{A} \times \mathrm{A} \\
\mathrm{A} \times \mathrm{A} \\
\mathrm{A} \times \mathrm{A}\end{array}$ & $\begin{array}{l}\text { K } 1114 \text { x K } 0607 \\
\text { K } 0307 \text { x K } 9423 \\
\text { K } \mathbf{0 6 0 7} \text { x K } \mathbf{0 9 1 1} \\
\text { K } 9162 \text { x K } 9423 \\
\text { DBW 14 x K 0424 }\end{array}$ \\
\hline Grain weight per spike (g) & $\begin{array}{l}\text { DBW } 14 \text { x K 0424** } \\
\text { K } 0607 \text { x K 0911*** } \\
\text { K } 1114 \text { x K } 0424 * * \\
\text { K } 9162 \text { × K 9423** } \\
\text { K } 0307 \text { x K 9423*** }\end{array}$ & $\begin{array}{l}\mathrm{L} \times \mathrm{L} \\
\mathrm{A} \times \mathrm{H} \\
\mathrm{H} \times \mathrm{L} \\
\mathrm{A} \times \mathrm{A} \\
\mathrm{H} \times \mathrm{A}\end{array}$ & $\begin{array}{l}\text { K } 0307 \text { × K } 9423 \\
\text { DBW } 14 \text { x K } 0424 \\
\text { K } 0607 \text { × K } 0911 \\
\text { K } 9162 \text { × K } 9423 \\
\text { K } 1114 \text { × K } 0424 \\
\end{array}$ \\
\hline Spike length $(\mathrm{cm})$ & $\begin{array}{l}\text { K } 9533 \text { x K 1114** } \\
\text { K } 1114 \text { x K 0424** } \\
\text { K } 0424 \text { x K 9423*** } \\
\text { K } 1114 \text { x DBW 14** } \\
\text { DBW 14 x K 0607** }\end{array}$ & $\begin{array}{l}\mathrm{H} \times \mathrm{A} \\
\mathrm{A} \times \mathrm{L} \\
\mathrm{L} \times \mathrm{L} \\
\mathrm{A} \times \mathrm{L} \\
\mathrm{L} \times \mathrm{H} \\
\end{array}$ & $\begin{array}{l}\text { K } 9162 \text { x K } 0307 \\
\text { K } 9533 \text { x K } 0307 \\
\text { K } 0607 \text { x K } 0911 \\
\text { DBW 14 x K 0607 } \\
\text { K } 0607 \text { x K } 0307 \\
\end{array}$ \\
\hline Biological yield per plant (g) & $\begin{array}{l}\text { DBW14 x K 0424** } \\
\text { K } 1114 \text { x NW 2036** } \\
\text { K } 9162 \text { x K 9423** } \\
\text { K } 1114 \text { x K } 0424 * * \\
\text { K } 0607 \text { x K 0911** } \\
\end{array}$ & $\begin{array}{l}\text { A } \times \text { A } \\
\mathrm{L} \times \mathrm{L} \\
\mathrm{A} \times \mathrm{H} \\
\mathrm{L} \times \mathrm{A} \\
\mathrm{A} \times \mathrm{A}\end{array}$ & $\begin{array}{l}\text { DBW } 14 \text { x K } 0424 \\
\text { K } 0911 \text { x K } 0307 \\
\text { K } 0607 \text { x K } 0911 \\
\text { K } 1114 \text { x NW } 2036 \\
\text { K } 9162 \text { x K } 9423 \\
\end{array}$ \\
\hline Harvest index (\%) & $\begin{array}{l}\text { K } 1114 \text { x K 0424** } \\
\text { DBW } 14 \text { x K } 9423 * * \\
\text { K } 9162 \text { x K } 9423 * * \\
\text { K } 1114 \text { x NW } 2036 * * \\
\text { K } 9533 \text { x K 0307** } \\
\end{array}$ & $\begin{array}{l}\mathrm{H} \times \mathrm{L} \\
\mathrm{L} \times \mathrm{A} \\
\mathrm{A} \times \mathrm{A} \\
\mathrm{H} \times \mathrm{H} \\
\mathrm{L} \times \mathrm{H}\end{array}$ & $\begin{array}{l}\text { DBW } 14 \times \text { K } 9423 \\
\text { K } 1114 \times \text { K } 0424 \\
\text { K } 9162 \times \text { K } 9423 \\
\text { K } 9533 \times \text { K } 0307 \\
\text { DBW } 14 \text { x K } 0424 \\
\end{array}$ \\
\hline 1000 grain weight $(\mathrm{g})$ & $\begin{array}{l}\text { DBW } 14 \text { x K 0424** } \\
\text { K } 0424 \text { x NW 2036** } \\
\text { K } 1114 \text { x NW 2036** } \\
\text { K 0607 x K 0911** } \\
\text { K } 9162 \text { x K } 9423 * * \\
\end{array}$ & $\begin{array}{l}\mathrm{L} \times \mathrm{A} \\
\mathrm{A} \times \mathrm{A} \\
\mathrm{L} \times \mathrm{A} \\
\mathrm{H} \times \mathrm{A} \\
\mathrm{L} \times \mathrm{L}\end{array}$ & $\begin{array}{l}\text { K } 1114 \text { x NW } 2036 \\
\text { K } 0424 \text { x NW } 2036 \\
\text { K } 0307 \text { x K } 9423 \\
\text { K } 0607 \text { x K } 0911 \\
\text { DBW } 14 \text { x K } 0424 \\
\end{array}$ \\
\hline Spike density & $\begin{array}{l}\text { DBW } 14 \text { x K 0424** } \\
\text { DBW } 14 \text { x NW 2036** } \\
\text { K } 1114 \text { x K 0424** } \\
\text { K } 0911 \text { x K } 0307 * * \\
\text { K } 0424 \text { x K } 0307 * * \\
\end{array}$ & $\begin{array}{l}\mathrm{H} \times \mathrm{L} \\
\mathrm{L} \times \mathrm{A} \\
\mathrm{A} \times \mathrm{L} \\
\mathrm{A} \times \mathrm{L} \\
\mathrm{L} \times \mathrm{L}\end{array}$ & $\begin{array}{l}\text { DBW } 14 \text { x NW } 2036 \\
\text { DBW } 14 \text { x K 0424 } \\
\text { K } 1114 \text { x K } \mathbf{0 4 2 4} \\
\text { K } 1114 \text { x K } 0911 \\
\text { DBW } 14 \text { x K } 0911 \\
\end{array}$ \\
\hline Protein content (\%) & $\begin{array}{l}\text { K } 0607 \text { x K 0307*** } \\
\text { K } 0607 \text { x K 0911*** } \\
\text { DBW } 14 \text { x K 9423** } \\
\text { DBW } 14 \text { x K 0424** } \\
\text { DBW } 14 \text { x K } 0607 * *\end{array}$ & $\begin{array}{l}\mathrm{H} \times \mathrm{H} \\
\mathrm{H} \times \mathrm{H} \\
\mathrm{A} \times \mathrm{H} \\
\mathrm{A} \times \mathrm{H} \\
\mathrm{A} \times \mathrm{H}\end{array}$ & $\begin{array}{l}\text { K } 0607 \text { x K } 0911 \\
\text { K 0607 x K } 0307 \\
\text { K 0424 x K } 9423 \\
\text { DBW } 14 \text { x K 0424 } \\
\text { DBW 14 x K 9423 }\end{array}$ \\
\hline Grain yield per plant $(\mathrm{g})$ & $\begin{array}{l}\text { DBW } 14 \text { x K 0424*** } \\
\text { K } 9162 \times \text { K } 9423 * * \\
\text { K } 1114 \times \text { K 0424** } \\
\text { K } 1114 \times \text { xW 2036** } \\
\text { K } 9533 \times \text { K 0307** } \\
\end{array}$ & $\begin{array}{l}\mathrm{L} \times \mathrm{L} \\
\mathrm{A} \times \mathrm{A} \\
\mathrm{A} \times \mathrm{L} \\
\mathrm{A} \times \mathrm{L} \\
\mathrm{L} \times \mathrm{H}\end{array}$ & $\begin{array}{l}\text { DBW } 14 \text { × K } 0424 \\
\text { K } 9162 \times \text { K } 9423 \\
\text { K } 9533 \times \text { K } 0307 \\
\text { K } 1114 \times \text { K } 0424 \\
\text { K } 1114 \times \text { NW } 2036 \\
\end{array}$ \\
\hline
\end{tabular}


Table. 5 continued

\begin{tabular}{|c|c|c|c|}
\hline Character & Good specific combiner & gca effect of parent & $\begin{array}{l}\text { Superior crosses on the } \\
\text { basis of per se } \\
\text { performance }\end{array}$ \\
\hline $\begin{array}{l}F_{2} S \\
\text { Number of effective tillers per } \\
\text { plant }\end{array}$ & $\begin{array}{l}\text { K } 1114 \text { x K 0911** } \\
\text { DBW } 14 \text { x K 0424** } \\
\text { K } 1114 \text { NW 2036** } \\
\text { K } 9533 \text { x K 0307** } \\
\text { K } 9533 \text { x K 9423** }\end{array}$ & $\begin{array}{l}\mathrm{H} \times \mathrm{H} \\
\mathrm{L} \times \mathrm{H} \\
\mathrm{H} \times \mathrm{H} \\
\mathrm{A} \times \mathrm{A} \\
\mathrm{A} \times \mathrm{A}\end{array}$ & $\begin{array}{l}\text { K } 1114 \text { x K } 0911 \\
\text { K } 9533 \times \text { K } 0307 \\
\text { K } 1114 \text { x NW } 2036 \\
\text { K } 9533 \text { x K } 0607 \\
\text { K } 9533 \times \text { K } 9423\end{array}$ \\
\hline Number of spikelets per spike & $\begin{array}{l}\text { K } 1114 \text { x K 0607** } \\
\text { K } 0607 \text { x K 0424** } \\
\text { K } 9533 \text { x K 1114** } \\
\text { DBW } 14 \text { x K 0424** } \\
\text { DBW } 14 \text { x NW 2036*** }\end{array}$ & $\begin{array}{l}\mathrm{L} \times \mathrm{A} \\
\mathrm{A} \times \mathrm{L} \\
\mathrm{H} \times \mathrm{L} \\
\mathrm{H} \times \mathrm{L} \\
\mathrm{H} \times \mathrm{A}\end{array}$ & $\begin{array}{l}\text { K } 0607 \text { x K } 0424 \\
\text { K } 1114 \text { x K } 0607 \\
\text { DBW } 14 \text { x K } 0307 \\
\text { DBW } 14 \text { x NW } 2036 \\
\text { K } 9533 \text { × K } 1114\end{array}$ \\
\hline Number of grains per spike & $\begin{array}{l}\text { K } 0307 \text { x NW 2036** } \\
\text { K } 0911 \text { x K 9423** } \\
\text { K } 1114 \text { x K 0424** } \\
\text { K } 9533 \text { x K } 0307 * * \\
\text { DBW } 14 \text { x K } 0607^{* * *}\end{array}$ & $\begin{array}{l}\mathrm{H} \times \mathrm{A} \\
\mathrm{A} \times \mathrm{A} \\
\mathrm{L} \times \mathrm{A} \\
\mathrm{A} \times \mathrm{H} \\
\mathrm{A} \times \mathrm{A}\end{array}$ & $\begin{array}{l}\text { K } 0307 \text { x NW } 2036 \\
\text { K } 0911 \times \text { K } 9423 \\
\text { K } 9533 \times \text { K } 0307 \\
\text { DBW } 14 \text { x K } 0424 \\
\text { K } 1114 \text { x K } 0424\end{array}$ \\
\hline Grain weight per spike (g) & $\begin{array}{l}\text { DBW } 14 \text { x K 0424*** } \\
\text { K } 0911 \text { x K 9423*** } \\
\text { K } 0607 \times \text { K 0911** } \\
\text { K } 0607 \text { x K 0424** } \\
\text { K } 9533 \text { x K 0307** }\end{array}$ & $\begin{array}{l}A \times A \\
A \times A \\
A \times A \\
A \times A \\
L \times H\end{array}$ & $\begin{array}{l}\text { K } 0307 \text { x NW } 2036 \\
\text { DBW } 14 \text { x K 0424 } \\
\text { K } 0911 \text { x K } 9423 \\
\text { K } 9533 \times \text { K } 0307 \\
\text { K } 0607 \text { x K } 0424 \\
\end{array}$ \\
\hline Spike length (cm) & $\begin{array}{l}\text { K } 0424 \text { x K 9423*** } \\
\text { K } 9533 \text { x K } 1114^{* *} \\
\text { K } 9533 \text { x DBW 14** } \\
\text { K } 1114 \text { x K 0607** } \\
\text { K } 0911 \text { x K } 9423^{* *}\end{array}$ & $\begin{array}{l}A \times L \\
L \times L \\
L \times A \\
L \times H \\
A \times L\end{array}$ & $\begin{array}{l}\text { K } 9162 \times \text { K } 0307 \\
\text { K } 0424 \times \text { K } 9423 \\
\text { K 9533 x DBW } 14 \\
\text { K } 9533 \text { × K } 0307 \\
\text { K } 0307 \text { x NW } 2036\end{array}$ \\
\hline Biological yield per plant (g) & $\begin{array}{l}\text { K } 9533 \times \text { K 0307*** } \\
\text { K } 0911 \text { x K 9423** } \\
\text { DBW } 14 \text { x K 0424** } \\
\text { K } 9162 \text { x K 9423** } \\
\text { K } 1114 \text { x NW 2036** }\end{array}$ & $\begin{array}{l}\mathrm{H} \times \mathrm{H} \\
\mathrm{H} \times \mathrm{H} \\
\mathrm{L} \times \mathrm{L} \\
\mathrm{H} \times \mathrm{H} \\
\mathrm{L} \times \mathrm{A}\end{array}$ & $\begin{array}{l}\text { K } 9533 \times \text { K } 0307 \\
\text { K } 0911 \times \text { K } 9423 \\
\text { K } 9162 \times \text { K } 9423 \\
\text { K } 9533 \times \text { K } 9423 \\
\text { K } 0911 \text { × K } 9423 \\
\end{array}$ \\
\hline Harvest index (\%) & $\begin{array}{l}\text { K } 1114 \text { x K 9423** } \\
\text { DBW } 14 \text { x K 0911*** } \\
\text { K 9533 x K 0911** } \\
\text { NW } 2036 \text { x K } 9423^{* *} \\
\text { K } 9162 \text { x K } 0607^{(N S)}\end{array}$ & $\begin{array}{l}\mathrm{H} \times \mathrm{A} \\
\mathrm{A} \times \mathrm{H} \\
\mathrm{L} \times \mathrm{H} \\
\mathrm{A} \times \mathrm{A} \\
\mathrm{A} \times \mathrm{A}\end{array}$ & $\begin{array}{l}\text { K } 1114 \text { x K } 9423 \\
\text { DBW } 14 \text { x K 0911 } \\
\text { K } 1114 \text { x K } 0911 \\
\text { K } 9533 \text { x K } 0911 \\
\text { DBW } 14 \text { x K } 0307\end{array}$ \\
\hline 1000 grain weight (g) & $\begin{array}{l}\text { K } 0424 \text { x NW 2036** } \\
\text { K } 9162 \text { x K 9423** } \\
\text { K 0424 x K 0307** } \\
\text { K } 0424 \text { x K } 9423 * * \\
\text { DBW } 14 \text { x K } 0424 * *\end{array}$ & $\begin{array}{l}\mathrm{A} \times \mathrm{H} \\
\mathrm{A} \times \mathrm{L} \\
\mathrm{A} \times \mathrm{H} \\
\mathrm{A} \times \mathrm{A} \\
\mathrm{H} \times \mathrm{A}\end{array}$ & $\begin{array}{l}\text { K } 0424 \text { x NW } 2036 \\
\text { K 0424 x K 0307 } \\
\text { K } 1114 \text { x NW } 2036 \\
\text { K } 9162 \text { x K } 0607 \\
\text { K } 9162 \text { x K } 9423\end{array}$ \\
\hline Spike density & $\begin{array}{l}\text { K } 9533 \text { x K } 0607 * * \\
\text { K } 9533 \times \text { K } 0911 * * \\
\text { DBW } 14 \text { x NW 2036*** } \\
\text { K } 9162 \text { x NW } 2036^{* *} \\
\text { K } 1114 \text { x K } 0307 * *\end{array}$ & $\begin{array}{l}\mathrm{H} \times \mathrm{L} \\
\mathrm{H} \times \mathrm{H} \\
\mathrm{H} \times \mathrm{A} \\
\mathrm{L} \times \mathrm{A} \\
\mathrm{H} \times \mathrm{L}\end{array}$ & $\begin{array}{l}\text { K } 9533 \times \text { K } 0911 \\
\text { K } 9533 \times \text { K } 0607 \\
\text { DBW } 14 \text { x NW } 2036 \\
\text { K } 9533 \text { x K } 9423 \\
\text { K } 1114 \text { x K } 0307 \\
\end{array}$ \\
\hline Protein content (\%) & $\begin{array}{l}\text { K } 0607 \text { x K 0307*** } \\
\text { K } 1114 \text { x K 0911*** } \\
\text { K } 9533 \text { x K } 1114 * * \\
\text { DBW } 14 \text { x K 0911** } \\
\text { K } 0911 \text { x K 0307*** }\end{array}$ & $\begin{array}{l}\mathrm{H} \times \mathrm{H} \\
\mathrm{A} \times \mathrm{H} \\
\mathrm{H} \times \mathrm{A} \\
\mathrm{L} \times \mathrm{H} \\
\mathrm{H} \times \mathrm{H}\end{array}$ & $\begin{array}{l}\text { K } 0607 \text { x K } 0307 \\
\text { K } 1114 \text { x K } 0911 \\
\text { K 0911 x K } 0307 \\
\text { DBW } 14 \text { x K } 0911 \\
\text { DBW } 14 \text { x NW } 2036 \\
\end{array}$ \\
\hline Grain yield per plant (g) & $\begin{array}{l}\text { DBW } 14 \text { x K 0424** } \\
\text { K } 9533 \text { x K 0307** } \\
\text { K } 0307 \text { x NW 2036** } \\
\text { K } 0911 \text { x K 9423** } \\
\text { K } 0607 \text { x K } 0424 * *\end{array}$ & $\begin{array}{l}\mathrm{L} \times \mathrm{L} \\
\mathrm{L} \times \mathrm{H} \\
\mathrm{H} \times \mathrm{A} \\
\mathrm{H} \times \mathrm{A} \\
\mathrm{A} \times \mathrm{L}\end{array}$ & $\begin{array}{l}\text { K } 9533 \text { x K } 0307 \\
\text { K } 0307 \text { x NW } 2036 \\
\text { K } 0911 \text { x K } 9423 \\
\text { DBW 14 x K 0424 } \\
\text { K } 9162 \text { × K } 9423\end{array}$ \\
\hline
\end{tabular}

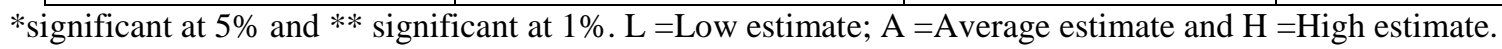


In present investigation, parent K 307 was identified good general combiner for grain yield and its component traits and K 0911 had also good general combining ability for grain yield and other quality traits over both generations in late condition, it means both parents possessed the desirable gene having good responsibility for produced good grain yield per plant of wheat and its quality traits under high temperature during grain filing period. Biological yield was the traits identified for selection with heat stress (Shah, 1998). The increase in productivity under late sown condition depends on the biomass attained by a genotype at the time of anthesis. The selection for high biomass yield should bring about positive improvement in grain yield and its associated characters. Thus the selection for biomass yield is one of the ways to improve the productivity in bread wheat.

For object to synthesize a dynamic population with most of the favorable gene accelerated by using of good general combiners for several characters, multiple crossing programme. Apart of conventional breeding approaches resting slowly upon additive or additive $\mathrm{x}$ additive type gene action, population improvement appears to be hopeful alternatives. Diallel selective mating system sounds to be good technique, which delays quick fixation of genes complexes, permits break down of linkage, general fostering of combination and concentration of desirable genes or gene groups into central gene pool by a series of multiple crosses. The sca represents the dominance and epistatic interaction, which can be related with heterosis. However, in selfpollinated crops like wheat, the additive $\mathrm{x}$ additive type of interaction component is fixable in later generations. Breeder's interest, therefore, vests in obtaining transgressive segregants through crosses and producing more potent homozygous lines. The superiority of hybrids might not indicate their ability to yield transgressive segregants, rather sca would provide satisfactory criteria (Jinks and Jones, 1958).

The estimate of specific combining ability ( $s c a$ ) revealed that out of 45 crosses, 18 crosses in $F_{1} \mathrm{~S}$ and 13 cross combinations in $\mathrm{F}_{2} \mathrm{~s}$ were identified good specific combiners for grain yield per plant (Table 3). In comparable study of top 5 crosses of each $F_{1}$ and $F_{2}$ generations, only two cross combinations revealed that good positive and significant sca effects. The highest positive and significant sca effects as well as their per se performance was exhibited by cross DBW $14 \mathrm{x}$ K 0424 and K 9533 x K 0307 in both generation for grain yield per plant. Whereas, other cross combination viz., K 9162 x K 9423, K 1114 x K 0424, K 1114 x NW 2036 in $F_{1}$ s and K 0307 x NW 2036, K 0911 x K 9423 K 0607 x K 0424 in $\mathrm{F}_{2} \mathrm{~S}$ were identified good specific combiner for grain yield per plant on basis of sca effects. Cross combination K 0607 x K 0307 was found good specific combiner for protein content over both generation on the basis of sca effects as well as their per se performance (table 5). Crosses K 1114 x K 0911, K 1114 x NW 2036 and DBW 14 for number of effective tillers per plant, K 9533 x K 1114 and DBW 14 x K 0424 for number of spikelets per spike, DBW 14 x K 0424 and K 0607 x K 0911 for grain weight per spike, K 9533 x K 1114 for spike length, DBW 14 x K 0424, K 1114 x NW 2036, K 9162 x K 9423 for high biological yield per plant, DBW $14 \times \mathrm{K} 0424$ for 1000 grain weight were also identified for super sca effects over both generations. As generally, these cross combinations were showed good yielding capacity, in most of the crosses, one of the parents involved was good combiner indicating they produced desirable segregants.

All the best cross combinations for grain yield per plant also showed an average to high sca effects for most of the yielding components. It is response to production of new materials in future breeding programme for recombining of desirable alleles of genes in the genotypes.

All the important crosses involving parents with high $\mathrm{x}$ average, average $\mathrm{x}$ average and average $\mathrm{x}$ poor general combiners indicated that nonadditive type of gene actions, which are unfixable in nature, were involved in selected cross combinations. The results of high sca effect due to high $\mathrm{x}$ higher reflect additive $\mathrm{x}$ additive types of gene action and superiority of 
favorable genes contributed by both parents, while high $\mathrm{x}$ average or lox $\mathrm{x}$ low combiners indicate the interaction of additive dominance and dominance $\mathrm{x}$ dominance types of gene action, respectively. In such condition will be arisen in study materials, bi-parental progeny selection suggested by (Andrus, 1963) may be used to get some good transgressive segregants from crosses involving high $\mathrm{x}$ high and high $\mathrm{x}$ poor combiners.

\section{Acknowledgement}

We are extremely thanks to Department of Genetics and Plant Breeding of C.S. Azad University of Agriculture and Technology, Kanpur for valuable suggestions and assistance provided during the course of investigation.

\section{References}

Ajmal, S., Khalid, I. and Rehman, A.U. 2011. Genetic analysis for yield and some yield traits in bread wheat (Triticum aestivum L.). J. Agri. Res., 49(4): 447-454.

Andrus, C.F. 1963. Plant breeding systems. Euphytica, 12:205-228.

Ankita, S., Anil, K., Ekhlaque, A., Swati and Jaiswal, J.P. 2012. Combining ability and gene action studies for grain yield, its components and quality traits in bread wheat (T. aestivum L. em Thell.). Electronic J. Plant Breeding, 3(4): 964972.

Ashadusjaman, M., Shamsuddoha, M., Alam, M.L. and Begum, M.O. 2012. Combining ability and gene action for different root characters in spring wheat. J. Environ. Sci. Resour., 5(2): 73-76.

Bikram, S. and Ahmad, B.A. 2008. Combining behavior of elite synthetic hexaploid wheat with staple wheat cultivar. J. Res. SKUAST-J., 7(2): 218-224.

Desai, S.A., Lohithaswa, H.C., Hanchinal, R.R., Patie, B.N., Kalappanavar, I.K. and Math, K.K. 2005. Combining ability for quantitative traits in bread wheat (Triticum aestivum L.). Indian J. Genetics and Plant Breeding, 65: 311-312.

Dubey, L.K., Sastry, E.V.D. and Sinha, K. 2001. Heterosis for yield and yield components in bread wheat (Triticum aestivum L.) under saline and normal environments. Annals of Arid Zone, 40: 57-60.

Griffings, B. 1956. Concept of general and specific combining ability in relation to diallel crossing system. Australian J. Biol. Sci., 9: 463-493.

Jensen, N.F. 1970. A diallel selective mating system for cereal breeding. Crop Sci., 10: 629-635.

Jinks, J.L. and Jones, R.M. 1958. Estimation of heterosis. Genetics, 43: 223-234.

Kapoor, E., Mandal, S.K. and Dey, T. 2011. Combining ability analysis for yield and yield contributing traits in winter and spring wheat combinations. J. Wheat Res., 3(1): 52-58.

Shah, M.A. 1998. Genetic studies for grain and temperature attributes in wheat (Triticum aestivum L.). The Indian J. Genetics and Plant Breeding, 61: 209-212.

Vanpariya, L.G., Chovatia, V.P. and Mehta, D.R. 2006. Combining ability studies in bread wheat (Triticum aestivum L.). National J. of Pl. Improvement, 8(2): 132137.

Wahid, A., Gelani, S., Ashraf, M. and Foolad, M. 2007. Heat tolerance in plant. An overview. Environ. Exp. Bot., 61: 199223.

\section{How to cite this article:}

Jaydev Kumar, S.K. Singh, Lokendra Singh, Mukul Kumar, Meera Srivastava, Jagbir Singh and Arun Kumar. 2017. Combining ability analysis for yield and its components in bread wheat (Triticum aestivum L.) under abiotic stress. Int.J.Curr.Microbiol.App.Sci. 6(3): 24-39. doi: https://doi.org/10.20546/ijcmas.2017.603.003 